\title{
Countrywide PV hosting capacity and energy storage requirements for distribution networks: the case of Switzerland
}

\author{
Rahul Gupta ${ }^{1}$, Fabrizio Sossan ${ }^{2}$, Mario Paolone ${ }^{1}$ \\ ${ }^{1}$ Distributed Electrical Systems Laboratory, EPFL, Switzerland, ${ }^{2}$ PERSEE, MINES \\ ParisTech - PSL, France
}

\begin{abstract}
Distributed photovoltaic (PV) generation is typically connected to power distribution grids, which are not designed to host a large amount of production if it is significantly larger than their nominal electricity demand. Given the prominent role of $\mathrm{PV}$ in energy transition pathways, modeling the existing power distribution infrastructure's constraints and limitations is key for its reliable techno-economical analysis and expansion.

As countrywide models of the distribution grids are, in general, not available, this paper first tackles the problem of estimating medium voltage (MV) distribution grids starting from publicly available datasets. It then proposes a method to estimate the PV generation hosting capacity of such grids and extend it through energy storage systems.

As a final contribution and ultimate objective, this paper proposes a method to derive cost-optimal plans for countrywide deployment of PV generation and energy storage systems considering the MV power distribution infrastructure's technical limitations. The distributed PV generation potential is modeled with high-spatially resolved capacity factors. Results are discussed using Switzerland as a case study.
\end{abstract}

Keywords: Synthetic networks, Photovoltaic, Battery sizing 


\section{Acronyms}

$\begin{array}{ll}\text { BESS } & \text { Battery Energy Storage System } \\ \text { CDF } & \text { Cumulative Distribution Function } \\ \text { DSO } & \text { Distribution System Operator } \\ \text { EHV } & \text { Extra High Voltage } \\ \text { ENTSOE } & \text { European Network of Transmission System Operators for Electricity } \\ \text { GCP } & \text { Grid Connection Point } \\ \text { GHI } & \text { Global Horizontal Irradiance } \\ \text { GIS } & \text { Geographical Information System } \\ \text { GW } & \text { GigaWatt } \\ \text { GWh } & \text { GigaWatthour } \\ \text { HV } & \text { High Voltage } \\ \text { LV } & \text { Low Voltage } \\ \text { kW } & \text { KiloWatt } \\ \text { MVA } & \text { MegaVoltAmpere } \\ \text { MV } & \text { Medium Voltage } \\ \text { MW } & \text { MegaWatt } \\ \text { OPF } & \text { Optimal Power Flow } \\ \text { PV } & \text { Photo-Voltaic } \\ \text { POA } & \text { Plane of Array } \\ \text { SOE } & \text { State-of-Energy } \\ \text { SD } & \text { Standard Deviation } \\ \text { TSO } & \text { Transmission System Operator } \\ \text { TWh } & \text { TeraWatthours }\end{array}$

\section{Introduction}

Photo-voltaic (PV) generation is experiencing a significant growth thanks to the decreasing costs of the installations and reduced carbon footprint [1]. In the period 2010-2019, PV has been the most deployed power source among renewables, with over $600 \mathrm{GW}$ of newly connected generation capacity [2].

Assessing the generation potential of distributed PV has attracted significant attention in the recent literature. For example, the work in [3] performs rule-based estimations and [4] uses a GIS to assess it for a large part of Europe considering the land availability. The considered spatial scales go from city to subcontinental levels, as in [5-10] and [11], respectively. 
The works discussed above focus on estimating the PV generation potential without considering the impact on the power distribution systems. Distributed PV generation, such as rooftop PV plants installed either on urban industry or rural environments, are typically connected to distribution grids, which, however, are designed to primarily deliver power to consumers and, as a matter of fact, can interface a limited amount of power generation. This limitation is due to the DSO's requirements to satisfy the physical constraints of the power grid assets. The amount of PV generation that a distribution grid can connect without violations of the grid constraints is called PV hosting capacity.

As power distribution systems are an important asset of the electrical infrastructure and upgrade costs to increase their generation hosting capacity are substantial [12], a reliable techno-economical assessment of the distributed PV generation potential should be done in conjunction with an accurate assessment of the PV hosting capacity of the existing distribution grids. Motivated by this reason, this paper considers the problem of estimating the PV generation potential at the scale of a country subject to the local distribution grids' limitations.

The PV hosting capacity of distribution grids is typically assessed for MV and LV distribution systems with probabilistic load flows applying the Monte Carlo method [13 16], or by less computationally intensive variations [17], and OPF models [18, 19]. Load flow- and OPF-based analyses require the knowledge of the grid topology, lines characteristics (length, physical parameters, buried/aerial type), and demand and PV generation profiles. Due to the large diversity of distribution grids in terms of topology and demand patterns, it is generally not possible to extend the results from a few known networks to the level of a country, which, depending on its size, might have thousands of MV distribution grids with different features.

As grid data are generally confidential, obtaining detailed distribution grid information for many networks to perform extensive load flows, is generally not an option. This challenge has inspired researchers to estimate grid topologies and characteristics from public data sets. For example, the works in [20 24] use data from existing grid models to estimate information of unobserved grids (i.e., supervised learning). On the other hand, the works [25] 28] used socio-economic data, like population density map and electricity demand, to generate distribution networks models without prior knowledge on the power grids (i.e., unsupervised).

In this paper, we investigate the PV hosting capacity of MV distribution 
grids for a whole country, using Switzerland as a case study. We consider MV networks because, according to [12], they account for the most significant part of the total grid upgrade costs when extending the PV generation hosting capacity. As grid data for the whole country are not available, we first propose an unsupervised method to infer their topology and characteristics starting from the publicly available locations of the EHV nodes and georeferenced energy demand data at high spatial resolution. With respect to existing unsupervised methods in 25] 28 described above, our method relies on less information, requiring only the location of the EHV substations and the spatial distribution of the demand. Then, we proceed by identifying the PV hosting capacity of each estimated grid with a tractable OPF based on linearized grid models, including also how to optimally deploy BESSs to increase the grids' PV hosting capacity. Finally, we determine the countrywide cost-optimal deployment of PV generation and BESSs to achieve a target level of PV installed capacity accounting for the spatial information on the capacity factor of PV generation.

Compared to the works in [1, 3] that report country-specific analyses of the PV potential and works in [9, 10, 29 31] that specifically refer to Switzerland, we estimate, for the first time in the literature, the PV generation potential for a whole country subject to the limitations of the existing distribution networks infrastructure. Compared to the works in [13-16] that evaluates the PV hosting capacity of small systems, we propose a method that can be extended to large areas, that estimates grid data and includes the deployment of BESSs to increase the PV hosting capacity.

In summary, the main contributions of this paper are:

- an unsupervised method to estimate MV grids starting from publicly available data;

- a tractable convex OPF model to estimate the PV hosting capacity of distribution grids, including cost-optimal BESSs siting and sizing to increase it;

- a tractable convex optimization problem to determine countrywide cost-efficient PV and BESS deployments plans to accommodate a target PV generation level accounting for the capacity factor of PV generation;

- the assessment of the optimal deployment plan for PV systems and 
BESSs in Switzerland to accommodate the PV generation target envisaged by the national energy strategy accounting for the constraints of the distribution grids.

The rest of this paper is organized as follows. Section 2 describes the methods to estimate the MV networks. We discuss the results of this process already in Section 2 so that, in the following sections, we can resort to these results for clarity and progress in the problem formulation. Section 3 describes the PV hosting capacity problem, including the sizing and siting of the BESSs. Section 4 presents and discusses the results of the countrywide optimal deployment of PV systems and BESSs Finally, Section 5 concludes this paper.

\section{Estimation of countrywide models of medium voltage power dis- tribution networks}

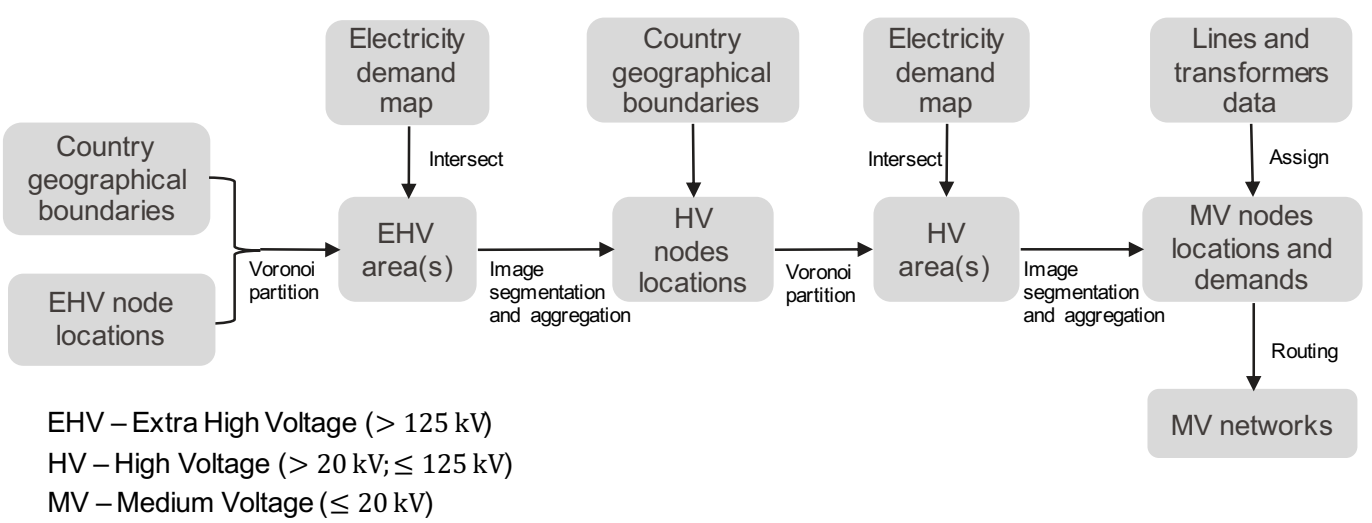

Figure 1: Flow chart for the estimation of countrywide models of medium voltage power distribution networks.

In this section, we describe the procedure to estimate the countrywide models of MV distribution grids. The procedure is graphically represented in Fig. 1 and summarized next. Starting from the locations of the EHV substations, we approximate the geographical region that each substation serves by partitioning the country with Voronoi diagrams (as described in subsection 2.1). We call these partitions EHV areas. Then, for each EHV area, we process the geographical distribution of the electricity demand to infer the position of the $\mathrm{HV}$ substations (subsection 2.2.2). By re-applying 
these two steps using the HV substation positions as input, we first identify the areas served by each HV substation, called HV areas. Finally, we find the locations of the MV substations (subsection 2.3). Once the location of the MV nodes are known, a routing scheme is used to estimate the topology and cable parameters of the relevant MV grids (subsection 2.4).

In order to exemplify the description of the proposed algorithms, in the following of this paper we specifically refer to the case of Switzerland.

\subsection{Identification of EHV areas}

$\mathrm{EHV} / \mathrm{HV}$ substations adapt the power grid voltage level from a value suitable for transmission over long distances to a more practical value for shortdistance transmission and more suitable to be transformed by secondary and tertiary substations to the final level at which electricity is consumed. As opposed to secondary and tertiary substations, the locations of the primary substation are available in public databases (e.g. [32]). We use them as the first step to infer the rest of the network.

In total, we consider 148 georeferenced EHV substations. The locations of the EHV nodes are from the dataset [33, that is derived from ENTSOE information. It was verified by visual inspection from aerial images that not all the locations from [33] correspond to the real ones, as also acknowledged on the ENTSOE website $e^{1}$. The inaccurate locations were corrected, when possible, by considering the locations reported in the collaborative dataset ${ }^{2}$, which were found accurate after being verified one by one on aerial images. The locations of the EHV nodes are shown in Fig. 2a.

Starting from the locations of the EHV substations, we apply Voronoi diagrams to approximate the region that each EHV node serves. Given an image and a collection of coordinates within that image, a Voronoi diagram (one per set of coordinates) is the closest locus of points to those coordinates. We use Voronoi diagrams because we reasonably assume that the electrical demand in a certain area is served by the closest substation. This modeling choice is also proposed in [26, 34, 36]. The result of the Voronoi partitioning is shown in Fig. 2b.

\footnotetext{
${ }^{1}$ https://www.entsoe.eu/data/map/

${ }^{2}$ https://en.wikipedia.org/wiki/List_of_EHV-substations_in_ Switzerland
} 


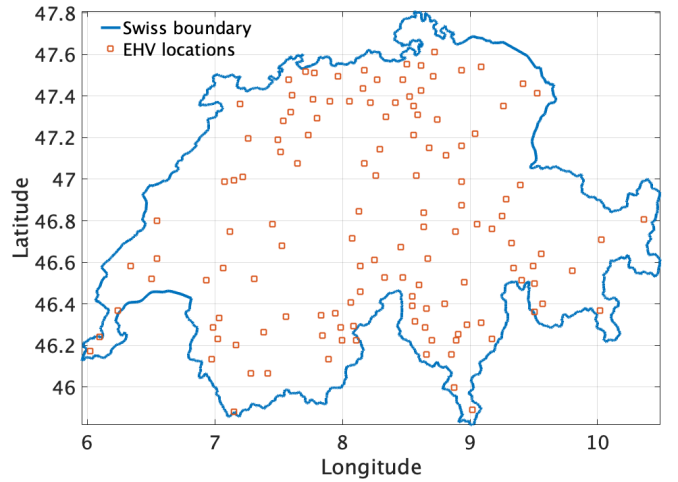

(a)

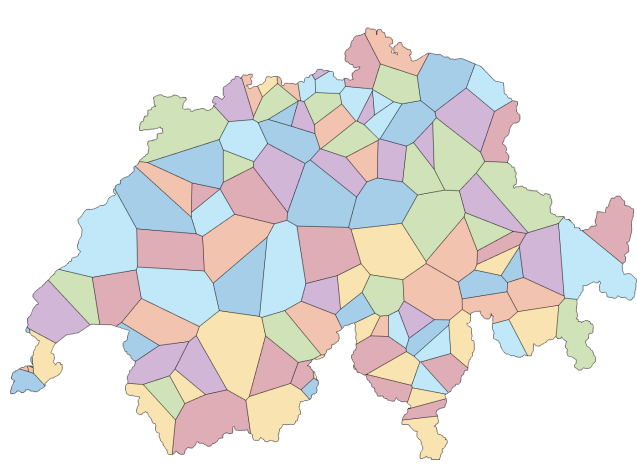

(b)

Figure 2: Identification of EHV areas: (a) locations of the considered 148 EHVs substations in Switzerland and (b) approximated regions served by each substation after Voronoi partitioning.

\subsection{Identification of the locations of the $H V / M V$ primary substations}

\subsubsection{Distribution of the electrical demand}

Power distribution systems were designed to deliver electricity to end customers. Therefore, we expect their topology and power ratings to reflect the geographical distribution of the demand for electricity. We leverage this notion and we start from the distribution of the electricity demand over the country to infer the topology of distribution systems. First, we estimate the distribution of the electricity demand as described next.

The work in [37] reports the statistics of the sectorial (industrial, commercial and residential) electricity consumption for each canton in Switzerland. This information gives already a comprehensive overview of the countrywide distribution of the electricity demand. However, since power distribution systems extend far deep into local regions, higher spatially resolved data are needed to estimate their topology. The Swiss Federal Office for Topography ${ }^{3}$ has mapped the heat demand for space heating and cooling for industrial, commercial, and residential buildings with a resolution of 100x100 meters. Since the heat demand follows the building distribution and that buildings are also large consumer of electricity (due to various electrical equipment, besides the obvious case of electric space heating [38, 39], that reinforces

\footnotetext{
${ }^{3}$ http://map.geo.admin.ch/
} 
Table 1: Composition of electricity demand in different sectors for Switzerland for 2014 37.

\begin{tabular}{|c|c|}
\hline Sector & Electricity demand (GWh) \\
\hline Residential & $1^{\prime} 333$ \\
\hline Commercial & $17^{\prime} 531$ \\
\hline Industrial & $1^{\prime} 028$ \\
\hline
\end{tabular}

the correlation among the two), we assume that the electricity and heat demands follow the same spatial distribution. With this assumption, we model the electricity demand map by rescaling heat demand map by appropriate coefficients such that its sum over space amounts to the total electricity demand for each sector reported in Table 1. The estimated countrywide electricity demand map is shown in Fig. 3a. Fig. 3b is an illustrative example of the electrical demand distribution within a single Voronoi cell. The geographical area each Voronoi cell is supplied by the substation corresponding to that cell.

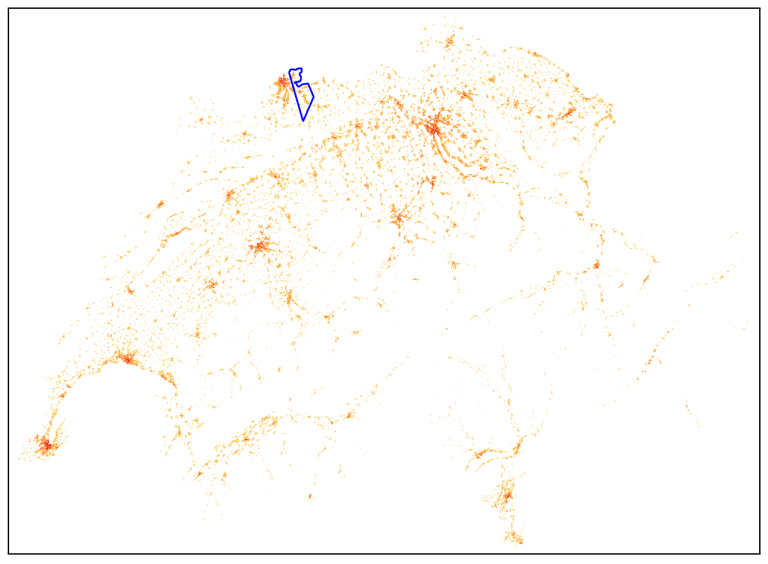

(a)

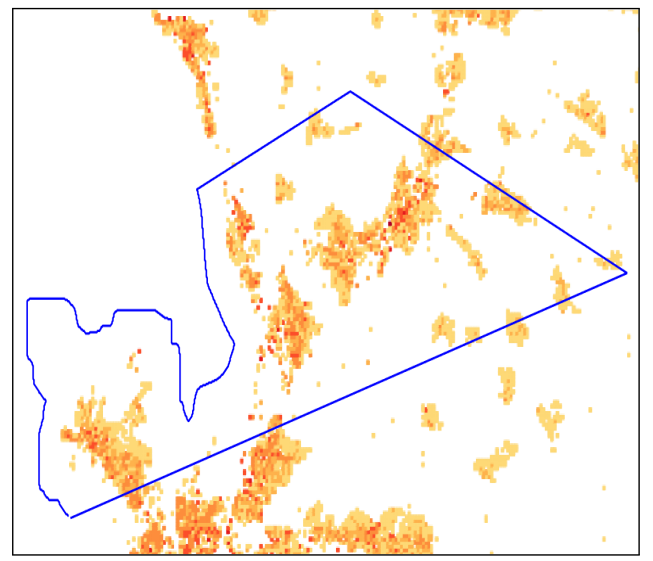

(b)

Figure 3: Estimated electricity demand map of: (a) Switzerland and (b) a single EHV area. The blue polygon refers to an EHV area obtained using Voronoi partition.

\subsubsection{Identification of the $H V / M V$ primary substations}

The location of the $\mathrm{HV} / \mathrm{MV}$ secondary substation is determined by analyzing the electrical demand map within each EHV area according to the 
following procedure.

1. Identify clusters with contiguous demand. To do so, we first derive a binary image from the electrical demand map, where Boolean true pixels denote non-zero electrical demand, and vice-versa. Then, to identify clusters with contiguous demand, we apply binary image segmentation, that partitions the input binary map into clusters containing pixels of the same kind (true or false) only. For the binary image segmentation, we use the bwboundaries Matlab function [40]. The result of this process for the example EHV area of Fig. 3b) is shown in Fig. 4a

2. On the one hand, clusters with total demand exceeding a pre-established threshold are recursively partitioned into smaller clusters using Algorithm 1. On the other hand, neighbour small clusters are aggregated until their total power demand reaches the threshold and so as to justify the presence of a secondary substation. The result of this step is illustrated in Fig. $4 \mathrm{~b}$. Threshold $\bar{L}$ in Algorithm 1 is an informed estimated computed as

$$
\frac{\text { average power demand }}{\text { number of EHV substation } \cdot 5}=\frac{63 \mathrm{TWh} / 8760 \mathrm{~h}}{148 \cdot 5} \approx 10 \mathrm{MW} \text {, }
$$

where 63 TWh is the total electricity demand in Switzerland in 2015 [41] and 5 is the estimated average number of $\mathrm{HV} / \mathrm{MV}$ nodes served by each $\mathrm{EHV} / \mathrm{HV}$ substation.

3. The location of each secondary substation is chosen at the geographical center of the convex envelope encompassing the respective aggregated cluster, as shown in Fig. $4 \mathrm{c}$.

Fig. 5a shows the distribution of the demand interfaced by the various primary substations, and the first row of Table 2 reports its mean and maximum value. It can be observed that, even if a static threshold of $10 \mathrm{MW}$ is used to generate the clusters, the demand within each cluster is finally spread around this value. On the one hand, larger values of the total demand happen because when merging multiple clusters, their aggregated demand might exceed the threshold. On the other hand, smaller values are because certain areas have low demand.

\footnotetext{
${ }^{4}$ Divide factor is chosen appropriately $(\leq 0.5)$ to obtain polygons with demands smaller than $L_{\text {thres }}$.
} 


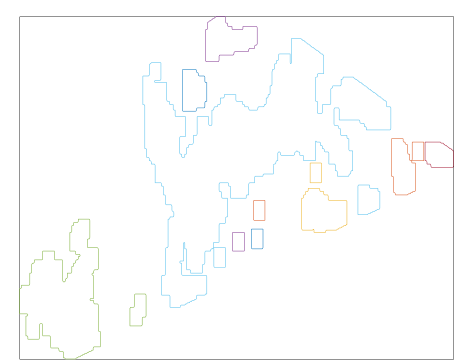

(a)

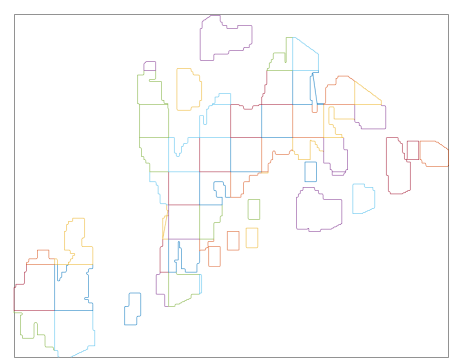

(b)

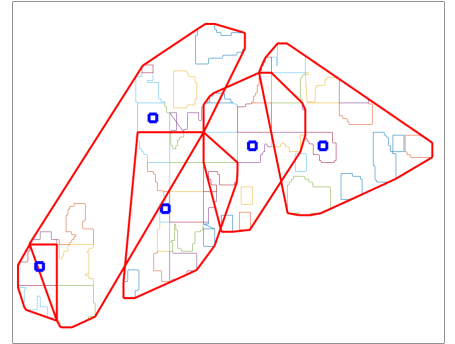

(c)

Figure 4: Identification of the substations locations: (a) the EHV area is divided into clusters, (b) large clusters are divided into smaller ones, (c) convex hull of the aggregated clusters (in red) and final locations of the HV substations (in blue).

\subsection{Identification of the $H V$ areas and $M V / L V$ secondary substations}

Once the locations of the primary substations are found, we apply the Voronoi partioning and cluster-aggregation procedures of subsections 2.1 and 2.2.2 to identify the HV areas and the MV/LV secondary substations. For the latter step, we use a threshold value for the total power within each cluster of $400 \mathrm{~kW}$. This value has been chosen because it is the average power rating of the nodes of the CIGRE benchmark grid for MV european systems [42]. The distribution of the demand interfaced by the secondary substation and its statistics are reported in Fig. $5 \mathrm{~b}$ and Table 2. Similarly to the previous case, the demand within each cluster is spread around the static threshold.

Table 2: Statistics on HV and MV substations

\begin{tabular}{|c|c|c|c|}
\hline Type & Number & Mean Demand & Max Demand \\
\hline HV substations & 776 & $9.3 \mathrm{MW}$ & $24.7 \mathrm{MW}$ \\
MV substations & 17,844 & $0.41 \mathrm{MW}$ & $0.97 \mathrm{MW}$ \\
\hline
\end{tabular}

Fig. 6a shows the identified locations of the substations for the example EHV area of Fig. 3b, where $5 \mathrm{HV} / \mathrm{MV}$ and $142 \mathrm{MV} / \mathrm{LV}$ substations were identified. This process is repeated for all EHV areas so as to estimate the locations of $\mathrm{HV} / \mathrm{MV}$ and $\mathrm{MV} / \mathrm{LV}$ substations for the whole country. For Switzerland, the model estimated $776 \mathrm{HV} / \mathrm{MV}$ nodes and 17'844 MV/LV, whose locations are shown in Fig 6b. 


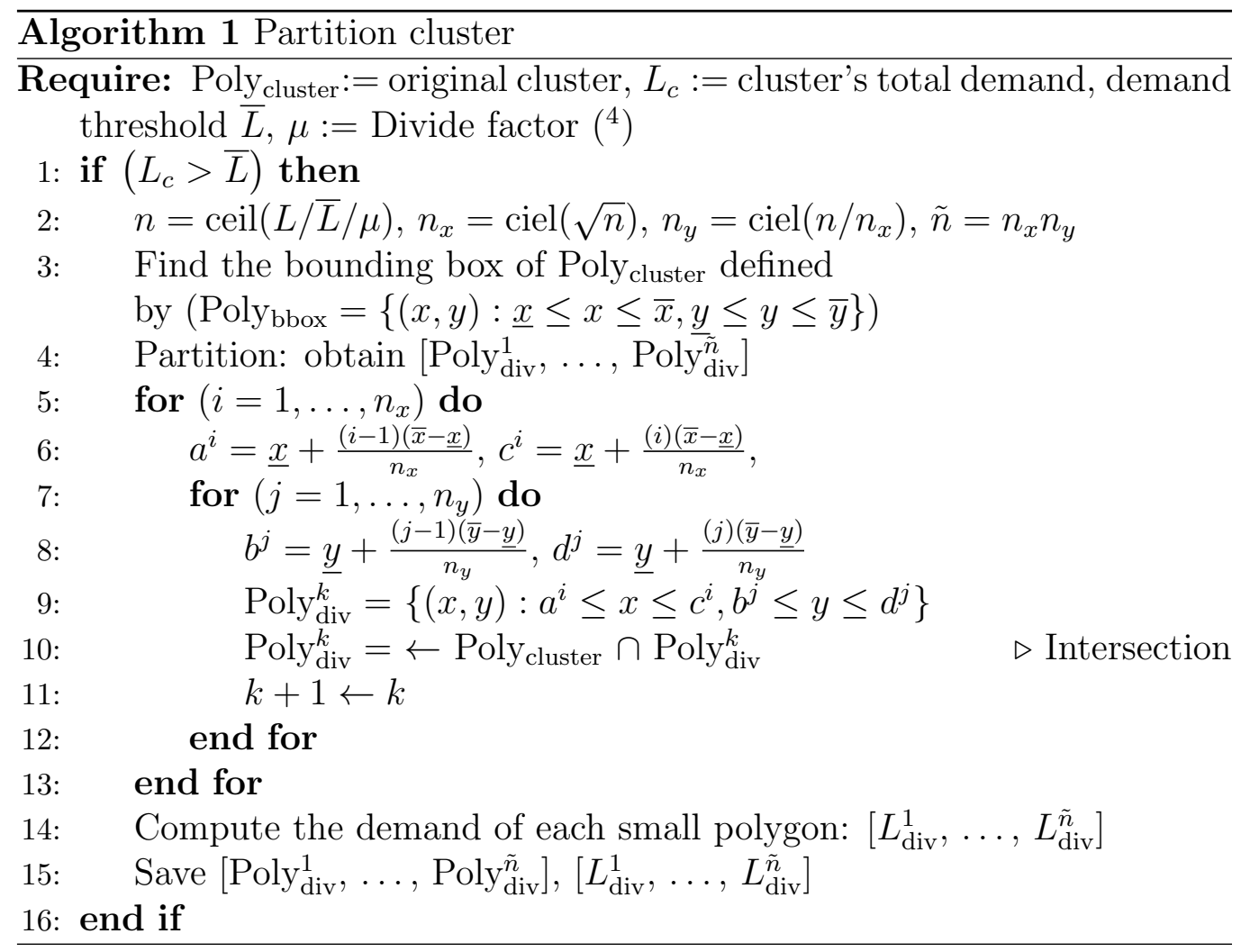

\subsection{Routing of medium voltage networks}

\subsubsection{Routing algorithm}

Once the locations of the MV substations are identified, we use a routing scheme to determine the connections and topologies of the corresponding grids. Several routing methods were proposed in the literature, as discussed in the review paper [43]. For example, the work in [44] uses a genetic algorithm and minimum spanning tree, works in [45 47] apply evolutionary algorithms such as simulated annealing and ant-colony. The work in [48, 49] proposes the branch-exchange method, and the work in [50] applies dynamic programming.

In this paper, we use the routing scheme based on the steepest gradient descent proposed in [51, 52] because of the faster convergence and increased tractability compared to the above-listed methods. The method accounts for the grid operational constraints on voltage magnitudes and lines ampacities. It enforces the radiality of the final system because the MV networks are 


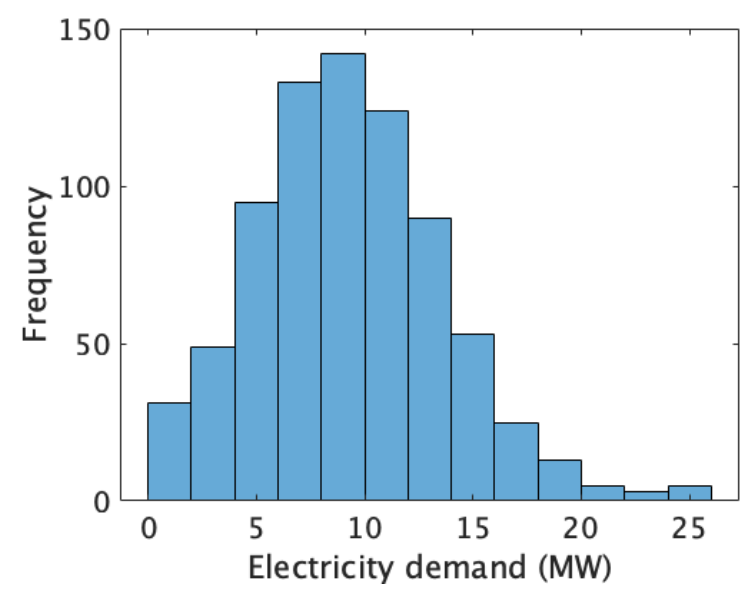

(a)

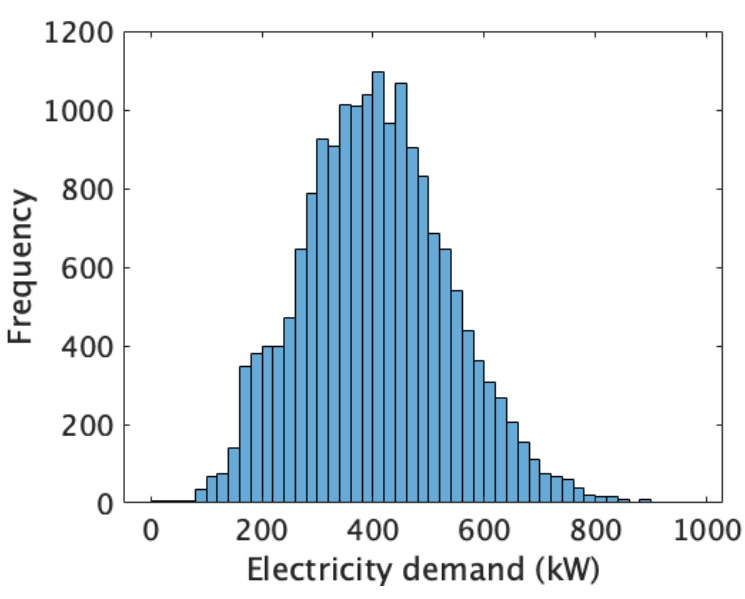

(b)

Figure 5: CDF plots of estimated electricity demands for (a) HV and (b) MV substations.

generally operated radially (as opposed to HV systems, that are typically meshed and operated as such). Although some MV network might have a meshed configuration (useful, e.g., for networks temporary operations in case of outages), they are usually operated radially to enable the effective operations of protection systems [53]. The method works by finding the grid topology that minimizes the capital cost of the grid, given by the investment cost for the power cables. In the routing scheme, we require voltage deviations to be up to $\pm 3 \%$ of the nominal voltage according to Swiss grid code [54]) and line currents below $80 \%$ of the respective cable ampacity, to reproduce a realistic scenario where grids operate with a safety margin from physical limits. The electrical characteristics of the lines and transformer used for the routing procedure are given in Tables 3 and 4 , respectively. The rating of transformer is assumed $150 \%$ of the total nominal demand to reflect a planning scenario where operators allow equipments to operate with a safety margin from their maximum ratings.

The routing scheme starts from a base topology where each substation node is connected to the 6 nearest ones (a value inspired from the work in [20] depicting an upper bound on the connections to/from a node in a typical power grid). Then, the following steps are performed:

1. run the routing scheme in Algorithm 2 by selecting high-ampacity type- 4 cables (from Table 3 ) for all the lines; 


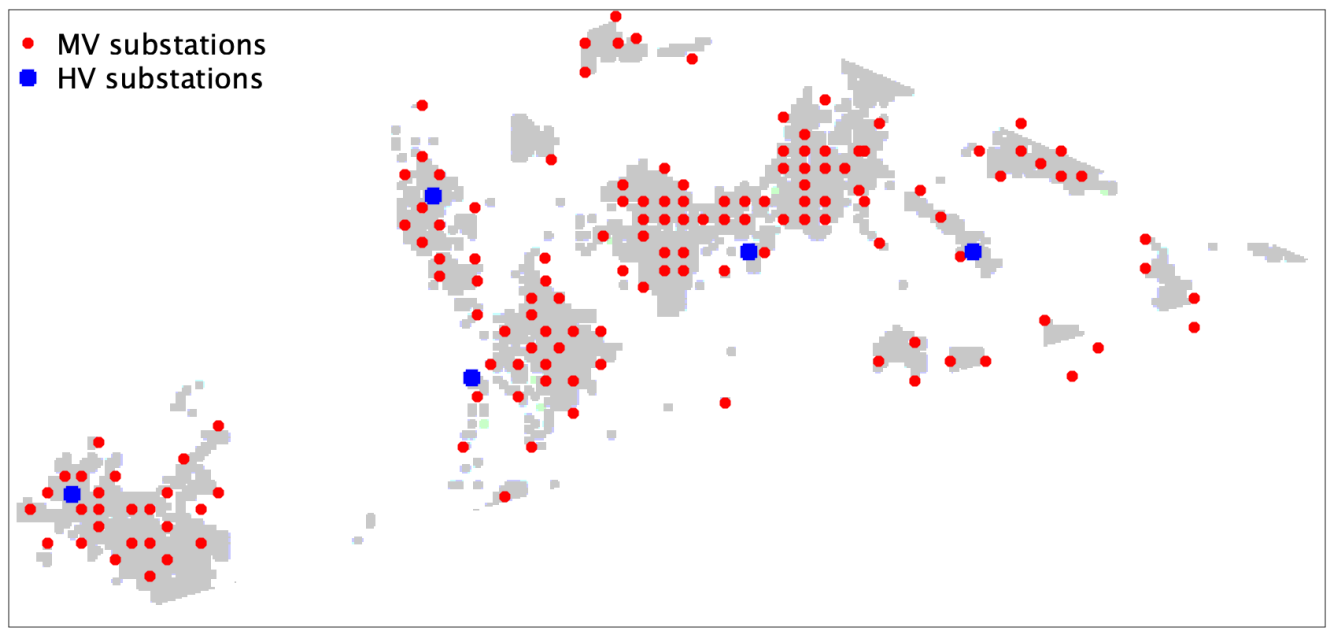

(a)

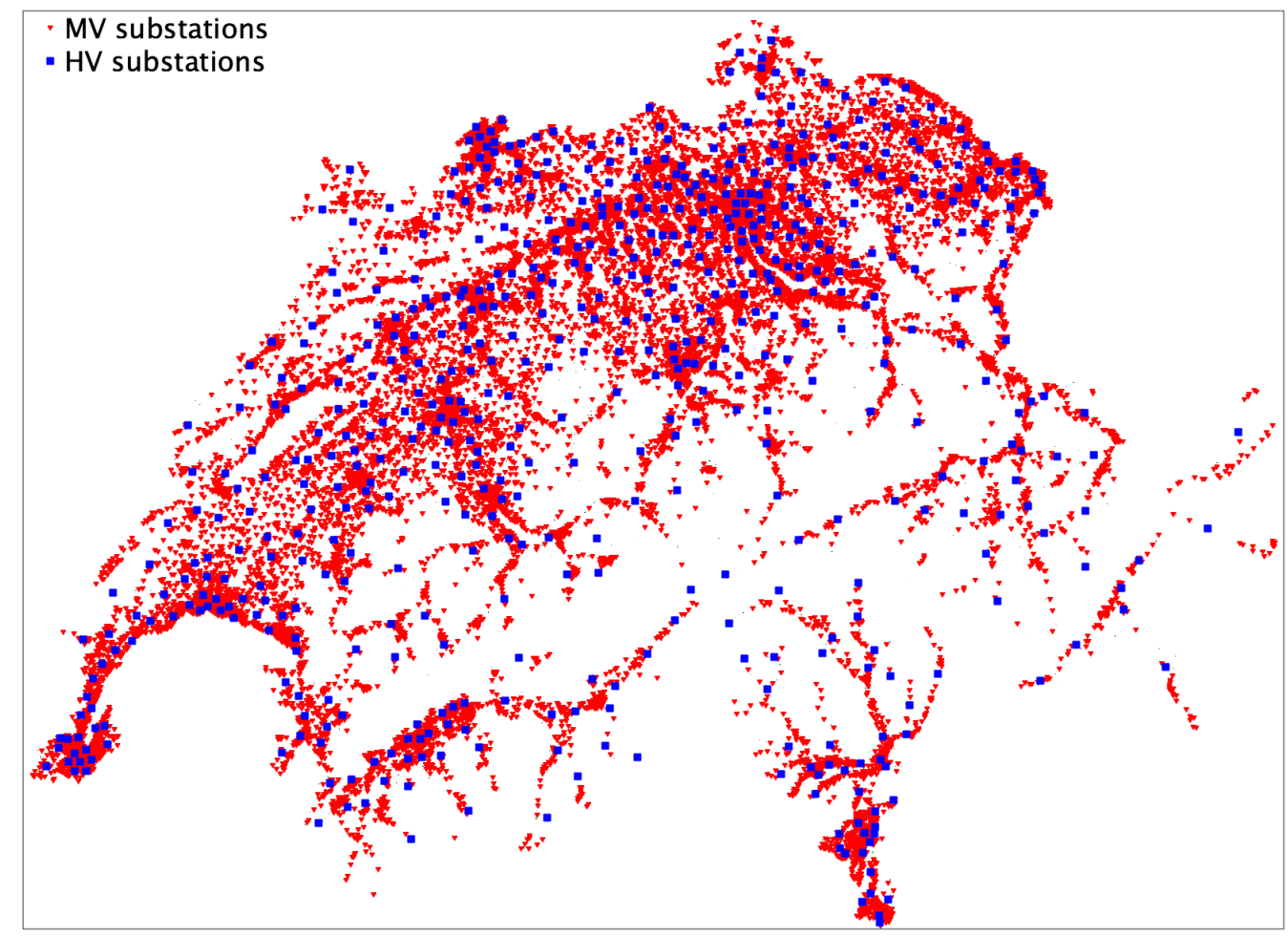

(b)

Figure 6: Identified HV/MV and MV/LV substations for (a) the example EHV area (5 and 142, respectively) and (b) Switzerland (776 and 17'844) 
2. replace the type- 4 cables (since they are most expensive ones according to their ampacity) with ones with lower ampacity according to the criterion reported in Table 5. For example, if the maximum line current in the first-stage routing is less than $10 \%$ of the type- 4 cable's ampacity, it is replaced with a type-1 cable. Once each single cable is replaced, we perform a load flow to verify voltage and current conditions and, if they are not satisfied, the original cable is restored.

Table 3: Cable ratings from a commercial source.

\begin{tabular}{|c|c|c|c|c|c|}
\hline $\begin{array}{c}\text { Cable } \\
\text { Type }\end{array}$ & $\begin{array}{c}\text { Section } \\
{\left[\mathrm{mm}^{2}\right]}\end{array}$ & $\begin{array}{c}\text { Resistance } \\
{[\mathrm{Ohm} / \mathrm{km}]}\end{array}$ & $\begin{array}{c}\text { Reactance } \\
{[\mathrm{Ohm} / \mathrm{km}]}\end{array}$ & $\begin{array}{c}\text { Capacitance } \\
{[\mu \mathrm{F} / \mathrm{km}]}\end{array}$ & $\begin{array}{c}\text { Ampacity } \\
{[\mathrm{A}]}\end{array}$ \\
\hline 1 & 50 & 0.495 & 0.13 & 0.19 & 228 \\
2 & 70 & 0.344 & 0.13 & 0.21 & 284 \\
3 & 95 & 0.248 & 0.12 & 0.23 & 346 \\
4 & 120 & 0.198 & 0.12 & 0.25 & 399 \\
\hline
\end{tabular}

Table 4: Transformer rating 42.

\begin{tabular}{|c|c|c|c|}
\hline $\begin{array}{c}\text { HV } \\
\text { voltage }[\mathrm{kV}]\end{array}$ & $\begin{array}{c}\text { MV } \\
\text { voltage }[\mathrm{kV}]\end{array}$ & $\begin{array}{c}\text { Short-circuit } \\
\text { impedance }[\text { Ohms] }\end{array}$ & $\begin{array}{c}\text { Power } \\
\text { rating [MVA] }\end{array}$ \\
\hline 110 & 20 & $0.016+\mathrm{j} 1.92$ & 25 \\
\hline
\end{tabular}

Table 5: Replacement scheme for lines.

\begin{tabular}{|c|c|}
\hline Current range (pu) & Cable type \\
\hline $0<0.1$ & 1 \\
$0.1 \leq 0.2$ & 2 \\
$0.2 \leq 0.4$ & 3 \\
\hline
\end{tabular}

Figure 7 shows the step-by-step routing results for an example EHV area. Fig. $7 \mathrm{~b}$ shows the initial routing, which is obtained by connecting each node with the nearest 6 nodes. Fig $7 \mathrm{c}$ shows an intermediate stage of the routing, where some of the redundant lines have been removed. The final topology is shown in Fig. 7d, where the color of the lines denotes their ampacities. Algorithm 2 works by iteratively removing the expensive (i.e., long) lines 


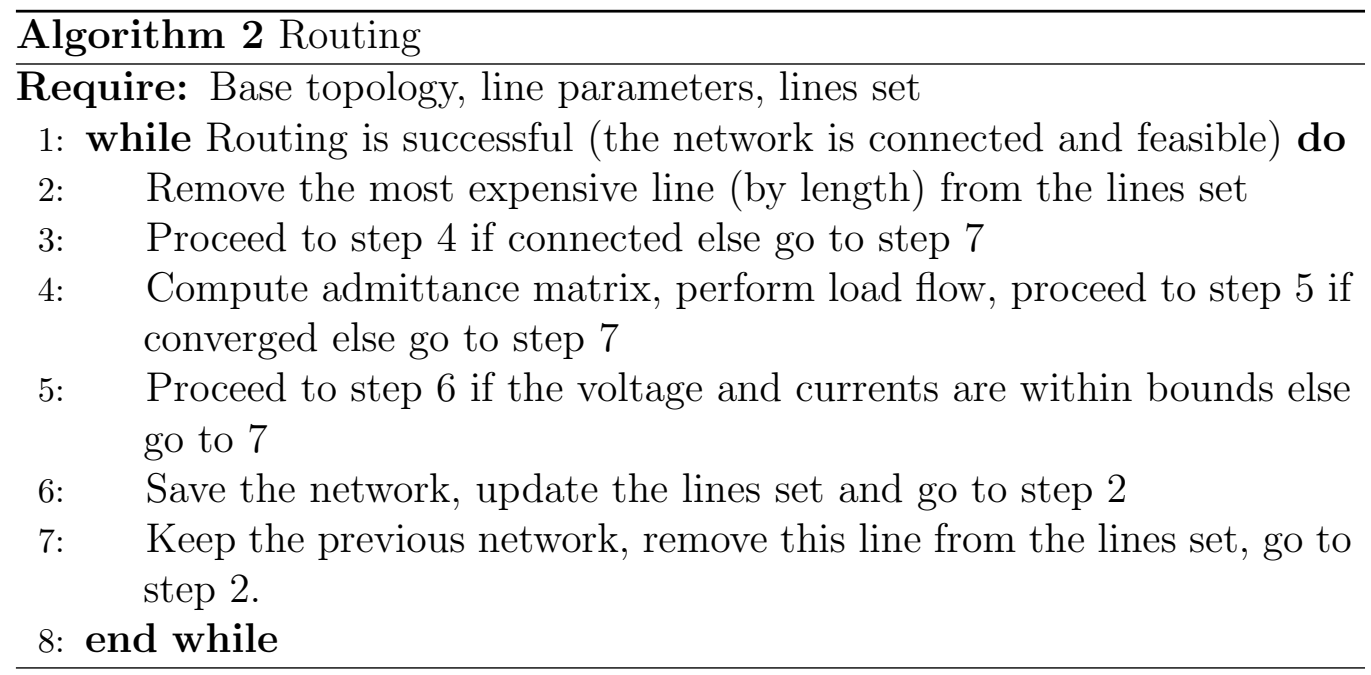

to minimize the cost of grid routing. The routing cost, expressed in terms of the total lines length, is shown in Fig. $7 \mathrm{e}$ for an example grid. It features a decreasing value before reaching a steady value after 300 iterations. In this example, the initial and final iterations correspond to $67 \mathrm{~km}$ and $10.5 \mathrm{~km}$, respectively, of deployed lines.

\subsubsection{Re-routing unsuccessful networks}

In certain cases, the routing by algorithm 2 might fail. This happens when a subset of the nodes in the given region is very distant in space to the rest of the nodes requiring very long cables. It either results in violations on voltage and currents or convergence issues while solving load-flows or requires a meshed topology with single or multiple rings to be feasible. These networks are labelled as unsuccessful networks. To solve this issue, we propose a rerouting procedure, where we divide the region further using a clustering method. The steps are described in algorithm 3. An example is shown in Fig. 8, where on the left figure, we see a meshed network to enable it to be routed due to current and voltage violations, whereas the right figure shows that the network is divided into two separate radial networks.

The final routing results for the example EHV region is shown in Fig. 9 , Statistics on the routed networks for the whole Switzerland are listed in the Table 6. The distributions of the nodal voltages and the lines currents are shown in Fig. 10 and denote that design requirements are met. More discussion on the validation of the estimated MV networks is presented in 


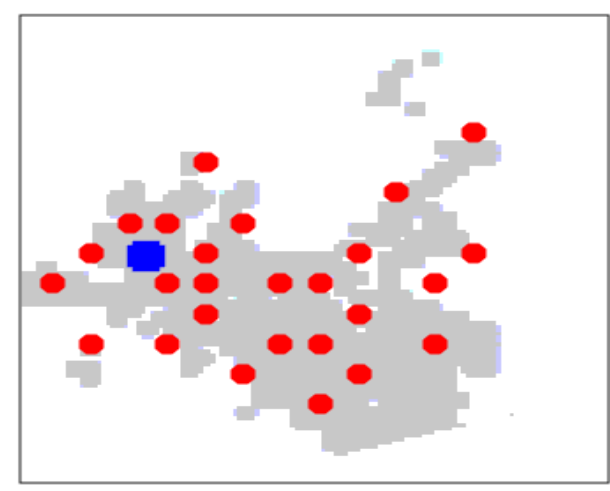

(a)

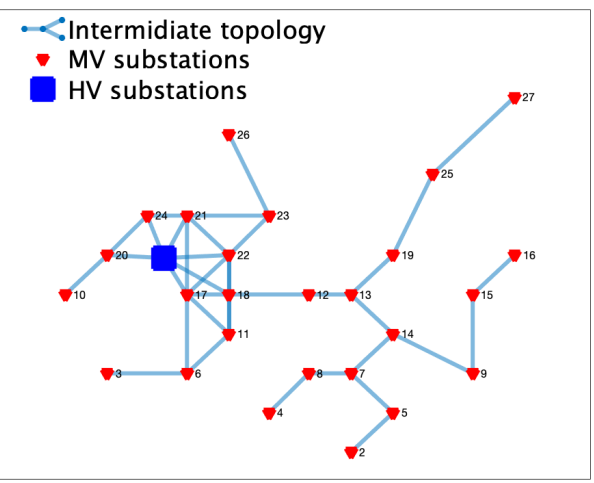

(c)

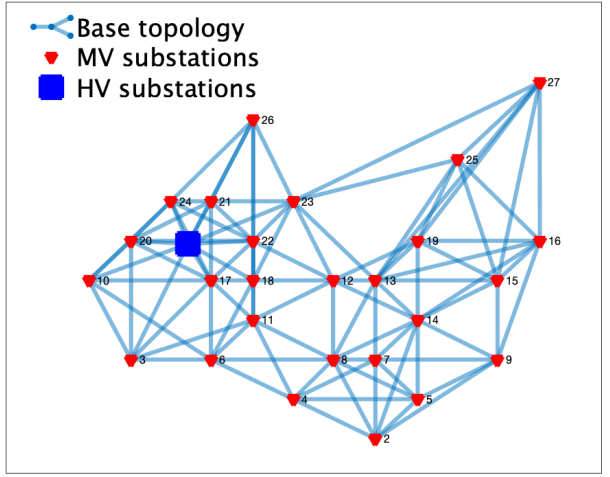

(b)

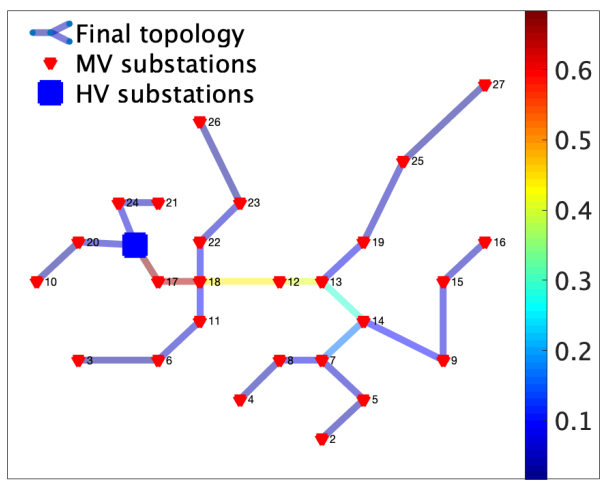

(d)

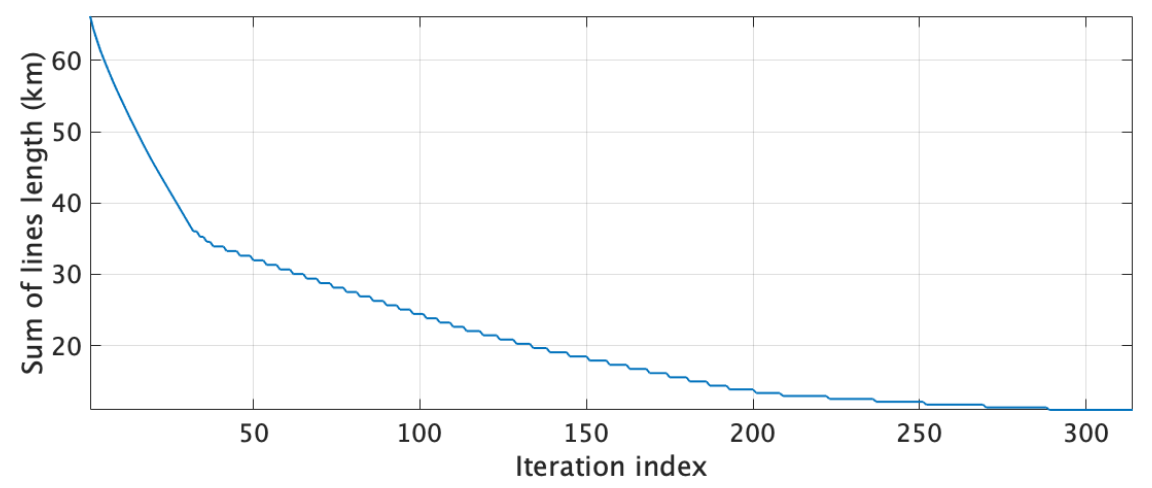

(e)

Figure 7: Routing procedure:(a) example EHV area with HV and MV substations, (b) highly-connected base topology, (c) meshed grid topology at an intermediate stage of the procedure, (d) final topology highlighting the current levels in the cables, and (e) total capital cost (expressed in $\mathrm{km}$ for length of cables used) as a function of the iteration. 


\begin{tabular}{|c|c|}
\hline Alg & ithm 3 Re-routing \\
\hline$\overline{\operatorname{Rec}}$ & ire: Substations' geographical locations \\
\hline & hile The network is connected and feasible do \\
\hline 2: & $\begin{array}{l}\text { Split the unsuccessful networks into two areas using } \mathrm{k} \text {-means } \\
\text { clustering with locations as features }\end{array}$ \\
\hline & $\begin{array}{l}\text { Place HV substations at the centroid of two areas, re-route both } \\
\text { the areas using algorithm } 2\end{array}$ \\
\hline 4: & Proceed to step 5 if network routing is successful else go to 2 \\
\hline 5: & Save the networks. \\
\hline & d while \\
\hline
\end{tabular}

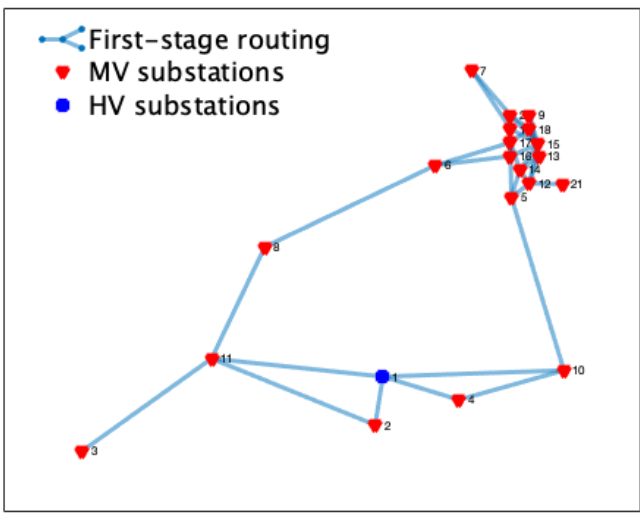

(a)

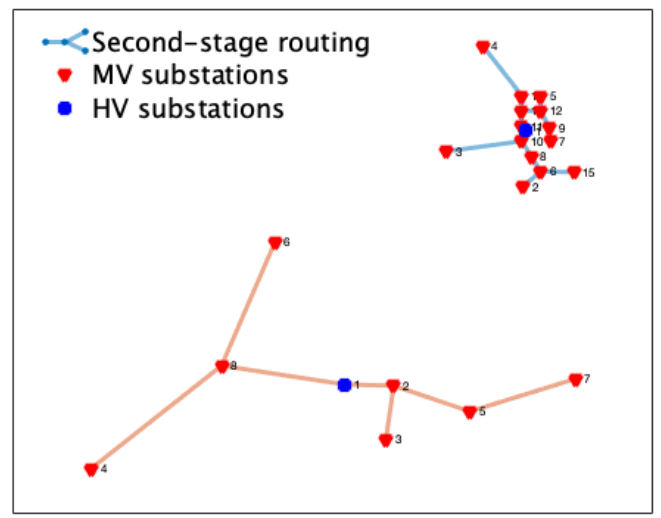

(b)

Figure 8: Re-routing: (a) routed network using algorithm 1 resulting in a meshed network, (b) routed network using algorithm 2 which divides it into two radial networks.

Appendix B.

\section{PV hosting capacity and energy storage requirements for power distribution networks}

The PV hosting capacity of a distribution grid is the maximum amount of PV generation that the grid can accommodate without violations of the its operational constraints. In this section, we describe the PV hosting capacity problem for distribution grids and, then, how to increase it with distributed energy storage systems [55]. Finally, we discuss the optimal deployment of PV power plants and BESSs to achieve the largest production at the 


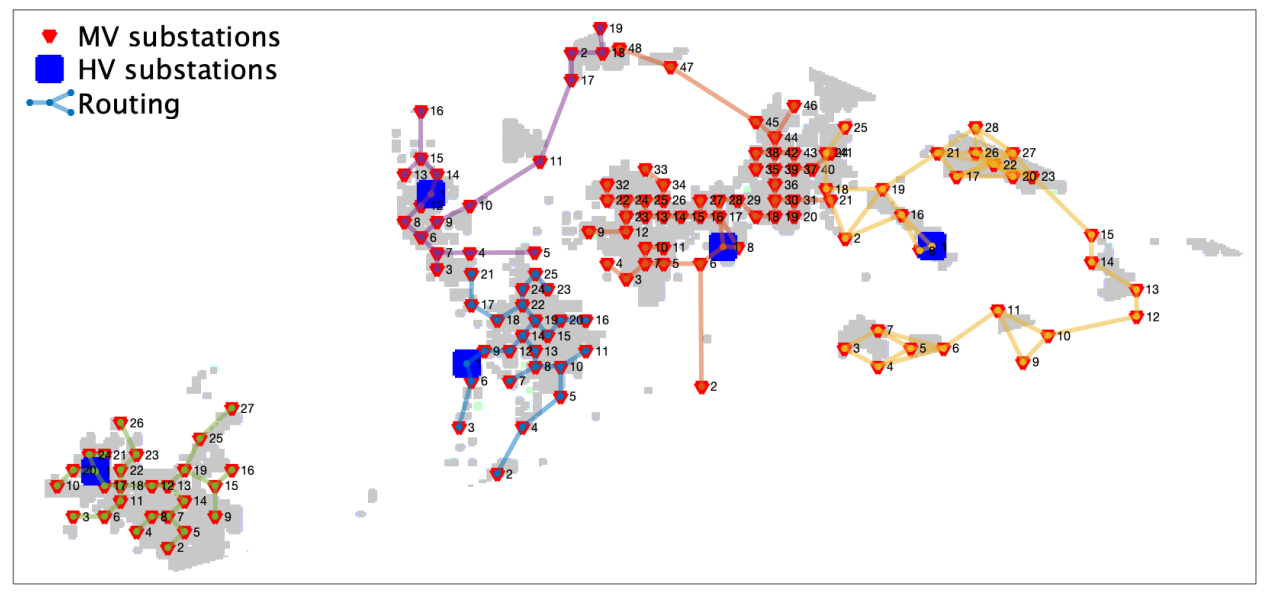

Figure 9: Routed MV networks for the example EHV area.

Table 6: Number of identified grid components.

\begin{tabular}{|c|c|}
\hline Equipment & Number of elements \\
\hline HV-MV transformers & 776 \\
MV-LV transformers & $17^{\prime} 844 \times 2$ (for redundancy) \\
MV cables and overhead lines & $1342.2 \mathrm{~km}$ \\
\hline
\end{tabular}

minimum cost for the whole country. We first discuss the input data that are used in the problem formulation.

\subsection{Input data}

\subsubsection{Capacity factor of $P V$ production}

PV capacity factors (total actual generation to the total generation at the nominal plant capacity over one year) for all locations across the country are used to compare the suitability for hosting PV generation. Capacity factors are from the PVGIS database [56] considering optimal panel locations (south-facing and $38^{\circ}$ tilt for the case of Switzerland). They are are based on satellite information at a $3 \times 3 \mathrm{~km}$ (at Nadir) resolution and are corrected for the shading induced by topographical features on the horizon. We query this information for the whole Switzerland with a resolution of $1.5 \times 1.5 \mathrm{~km}$. Figure 12a shows the distribution of the capacity factors across the country. It denotes variable values that can vary up to a factor of 3. 


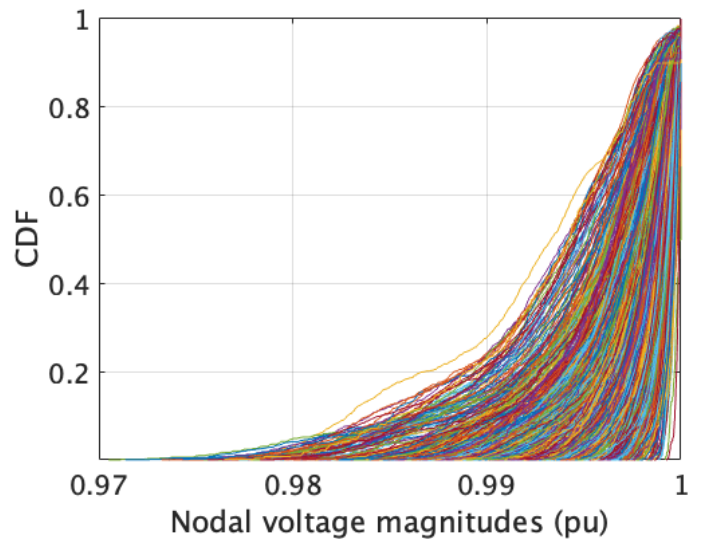

(a)

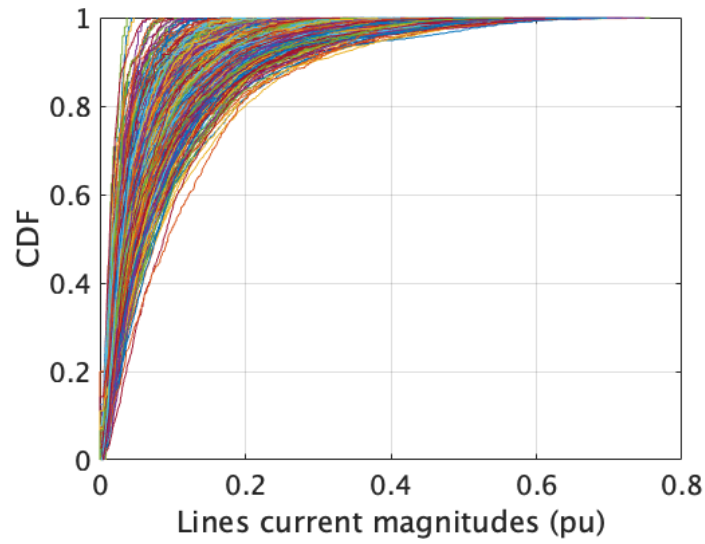

(b)

Figure 10: CDF plots (a) nodal voltages and (b) lines currents of estimated networks shown in different colors.

\subsubsection{Land-use constraints for $P V$ generation}

We evaluate land allocation to identify suitable locations for PV power plants. We use a 100x100 m resolution land-use map $5^{5}$ from the Swiss Federal Office for Topography, shown in Fig. 11, reporting settlement (residential, commercial, industrial and recreational) and agricultural areas. For the area corresponding to each MV grid, we consider that $10 \%$ of the settlement areas can host PV generation, for a total surface of $210 \mathrm{~km}^{2}$ for the whole country. Considering this available surface, the yearly capacity factors from PVGIS [56], and an average PV conversion efficiency of $15 \%$ in standard conditions [57, the yearly total PV generation for Switzerland with these assumption is of $33 \mathrm{TWh}$. Both the available area for PV deployment and total generation are in-line with the estimates reported in the existing literature [29 31, 58] as summarized in Table 7. Differences among the various estimations (more remarkably for PV generation) can be explained by different input data sets and methods, however they all seem to agree on the same order of magnitude. Fig. $12 \mathrm{~b}$ shows the distribution of the PV installed capacity potential (solely based on land availability) across all the MV grids of the country. Its mean and maximum values are 2 and $13.1 \mathrm{MW}$. The total PV installed capacity

\footnotetext{
5 https://map.geo.admin.ch/?layers=ch.bfs.arealstatistik-hintergrund\& lang=en\&topic=ech\&bgLayer=ch.swisstopo.pixelkarte-farbe
} 
Table 7: A comparison of the PV generation potential.

\begin{tabular}{|c|c|c|}
\hline Reference & Area $\left[\mathrm{km}^{2}\right]$ & Estimated PV generation [TWh] \\
\hline$[29$ & 328 & 17.86 \\
30 & 252 & 16.29 \\
{$[58$} & 485 & 41.32 \\
{$[3]$} & 267 & $24 \pm 9$ \\
\hline This work & 210 & 33 \\
\hline
\end{tabular}

potential with the above assumptions is of $30 \mathrm{GW}$. It is worth noting that larger capacity values are possible with higher usage of available land and PV conversion efficiency.

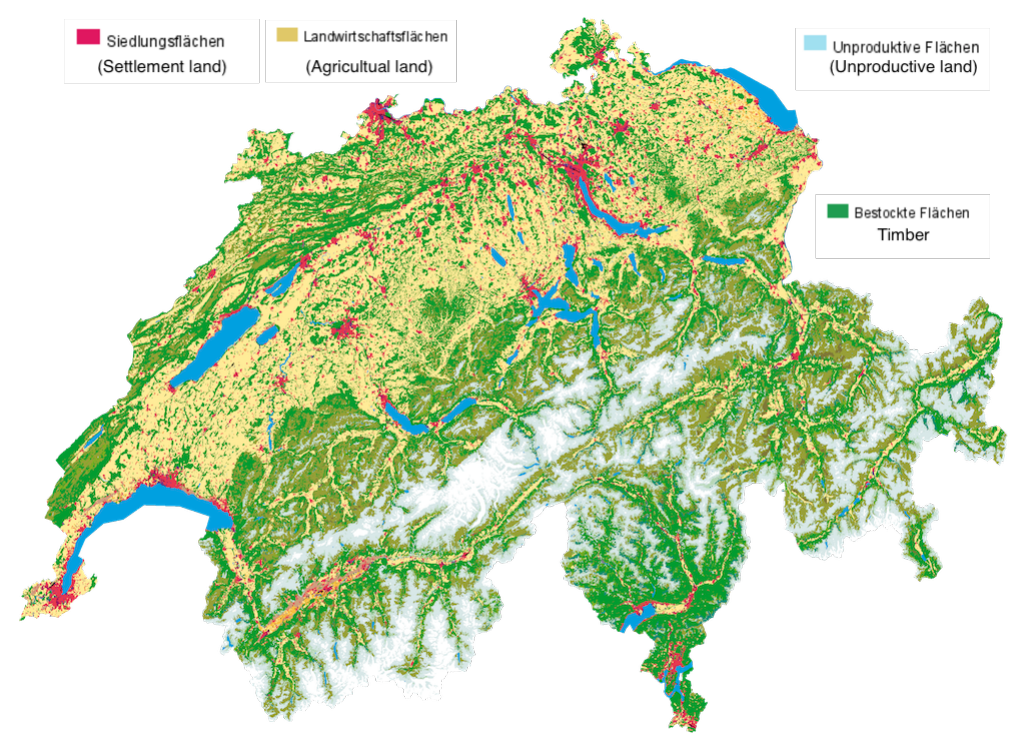

Figure 11: Simplified land-use map of Switzerland.

\subsubsection{Time series of the $P V$ generation and demand}

Solving the PV hosting capacity problem does require time series of PV generation and demand to model the loading conditions of the grid. We consider a scenario with high PV generation and low demand to reproduce cases where excess PV generation might cause violations of the grid constraints. In this respect, PV generation is modelled considering uniform clear-sky conditions over the whole power distribution network and considering the day 


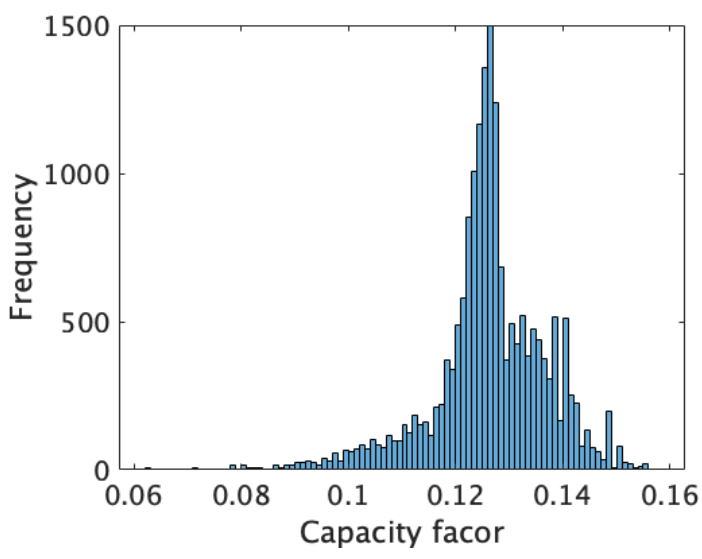

(a)

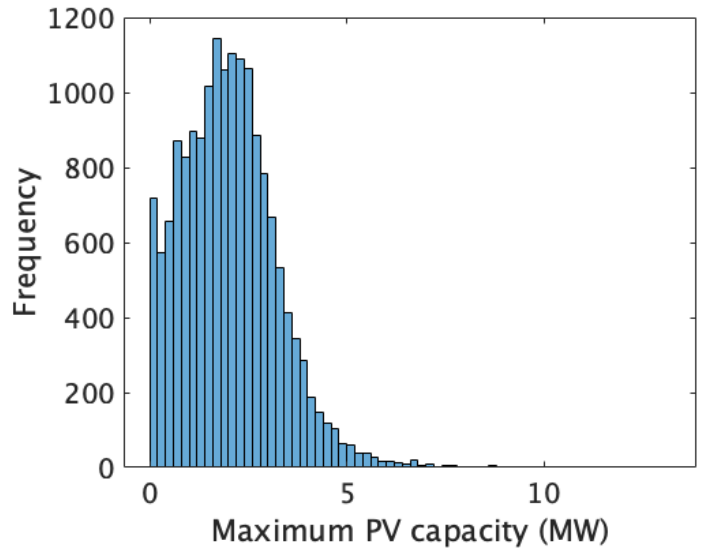

(b)

Figure 12: Distribution of (a) capacity factor and (b) maximum PV capacity per MV node due to land constraint.

of the year with the largest PV generation. We use a clear-sky model to compute the global-horizontal irradiance (GHI) as a function of the location, that we denote by $n$. The plane-of-array $(\mathrm{POA})$ irradiance $I_{t, n}\left(\mathrm{~kW} / \mathrm{m}^{2}\right)$ is determined by transposing the GHI as a function of the plant tilt and azimuth, and time of the day. The POA irradiance is finally converted to PV generation for a plant with $P^{\mathrm{pv}}$ capacity (in $\mathrm{kW}$ ) with the following model $g\left(t, n, P^{\mathrm{pv}}\right)$ :

$$
p_{t, n}^{\mathrm{pv}}=g\left(t, n, P^{\mathrm{pv}}\right)=I_{t, n}\left(1+\alpha\left(T_{t, n}^{\mathrm{air}}+\beta I_{t, n}-25\right)\right) P^{\mathrm{pv}}
$$

where $T_{t}^{\text {air }}$ is the air temperature $\left({ }^{\circ} C\right), \alpha=-0.0043$ and $\beta=0.038$ are empirical parameters as in [59] for open-rack PV plants.

Demand profiles are obtained by scaling the residential, commercial and industrial demand profiles specified in the CIGRE benchmark grid for MV systems [42], shown in Fig. 13a, for the coefficients extracted from the demand map computed in subsection 2.2. To reproduce a scenarios with dominant PV generation over the demand, we halve the nominal demand profile to reflect a day with low electricity consumption. We assume ideal correlation among the loads. Being the focus of the paper on modeling the impact of PV generation on the grid hosting capacity, modeling spatial diversity of the loads is not of special interest. We consider voltage- and frequency- 
independent loads. Figure $13 \mathrm{~b}$ shows the PV and the load profiles considered for the PV and battery sizing.

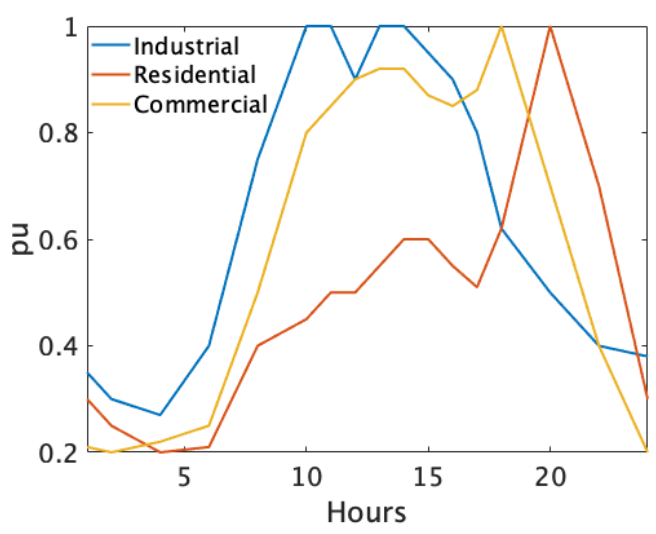

(a)

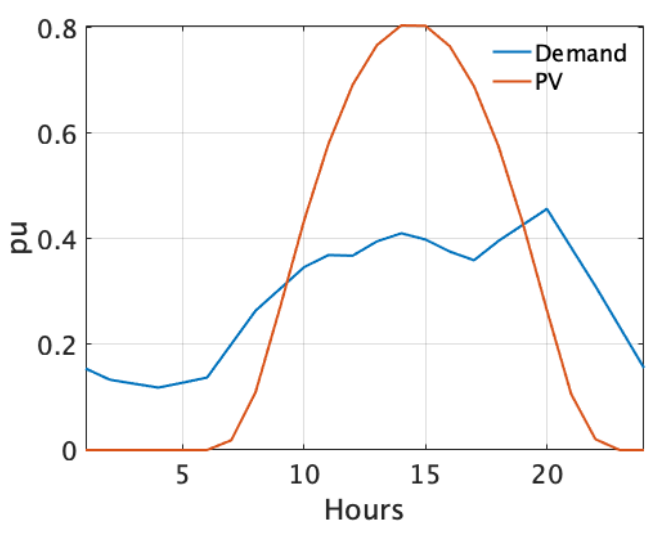

(b)

Figure 13: Demand and PV scenarios: (a) standard load profiles for different sector from [42], (b) scenario considered for the PV and battery sizing problem.

\subsection{The PV hosting capacity problem}

The objective of this problem is determining the maximum PV installed capacity that a grid can host at its nodes without violations of grid constraints. We consider a generic distribution grid with $N_{\text {bus }}$ nodes and $L$ lines with index $n \in \mathcal{N}=\left\{1, \ldots, N_{\text {bus }}\right\}$ and $l \in \mathcal{L}=\{1, \ldots, L\}$, respectively. The installed PV capacity at node $n$, that is an unknown of the problem, is denoted by $P_{n}^{\text {pv }}\left({ }^{6}\right)$. As discussed in Section 3.1.2, the installed capacity is limited by the land availability, so we say that $P_{n}^{\mathrm{pv}} \leq \overline{P_{n}^{\mathrm{pv}}}$, where the right-hand-side upper-bound is derived from the land availability map.

\subsubsection{Grid model}

In the following, bold-typeface notation refers to vectors. Active and reactive nodal injections at the various nodes of the grid are collected in vectors $\mathbf{p}_{t}, \mathbf{q}_{t}$. They are given by the difference between the nodal PV generation $\mathbf{p}_{t}^{\mathrm{pv}}, \mathbf{q}_{t}^{\mathrm{pv}}$ and demand $\mathbf{p}_{t}^{\text {load }}, \mathbf{q}_{t}^{\text {load }}$, when available. We assume that PV plants

\footnotetext{
${ }^{6}$ For generality, if a node cannot host PV generation, we can add in the following formulation a constraint of the kind $P_{n}^{\mathrm{pv}}=0$.
} 
operate at unitary power factor, so $\mathbf{q}_{t}^{\mathrm{pv}}=\mathbf{0}$, as typical for small/medium size PV plants. PV generation is computed by applying the model $g(\cdot)$ in (2). Vectors $\mathbf{v}_{t}$ and $\mathbf{i}_{t}$ collect the magnitudes of the nodal voltage and line current, respectively. These quantities, and the corresponding complex power at the grid connection point, are modelled as linear functions of the nodal injections and the voltage at the slack bus with a model based on sensitivity coefficients and described in 3.2.1. In the following, we denote the voltage and current linear model with the general notation $\mathbf{v}_{t}=v\left(\mathbf{p}_{t}, \mathbf{q}_{t}, \tilde{\mathbf{v}}_{0}\right)$ and $\mathbf{i}_{t}=c\left(\mathbf{p}_{t}, \mathbf{q}_{t}, \tilde{\mathbf{v}}_{0}\right)$, where $\tilde{\mathbf{v}}_{0}$ is the set of nodal voltage phasors for power-flow linearization. We model the grid with sensitivity coefficients which express the linearized dependency of nodal voltages, lines currents and grid losses as function of the nodal active and reactive power injections. The linear grid models for voltage, current and total grid losses are

$$
\begin{aligned}
& \mathbf{v}_{t}=v\left(\tilde{\mathbf{v}}_{0}, \mathbf{p}_{t}, \mathbf{q}_{t}\right)=\mathbf{A}_{\mathrm{t}}^{\mathbf{v}}\left[\begin{array}{l}
\mathbf{p}_{t} \\
\mathbf{q}_{t}
\end{array}\right]+\mathbf{b}_{t}^{\mathbf{v}} \\
& \mathbf{i}_{t}=c\left(\tilde{\mathbf{v}}_{0}, \mathbf{p}_{t}, \mathbf{q}_{t}\right)=\mathbf{A}_{\mathrm{t}}^{\mathbf{i}}\left[\begin{array}{l}
\mathbf{p}_{t} \\
\mathbf{q}_{t}
\end{array}\right]+\mathbf{b}_{t}^{\mathbf{i}} \\
& {\left[\begin{array}{l}
p_{t}^{\mathrm{gcp}} \\
q_{t}^{\mathrm{gcp}}
\end{array}\right]=s\left(\mathbf{p}_{t}, \mathbf{q}_{t}, \tilde{s}_{0}\right)=\mathbf{A}_{t}^{\mathrm{gcp}}\left[\begin{array}{l}
\mathbf{p}_{t} \\
\mathbf{q}_{t}
\end{array}\right]+\mathbf{b}_{t}^{\mathrm{gcp}}}
\end{aligned}
$$

where $\mathbf{A}$ and $\mathbf{b}$ are the linear mapping parameters obtained using the method in [60]. They are iteratively updated with newly sized battery and PV injections. An accuracy analysis of the modeled linear power flow is included in Appendix A. The symbols $p_{t}^{\text {gcp }}, q_{t}^{\text {gcp }}$ denotes the active and reactive power at the GCP. The nodal voltage magnitudes and line currents should be within allowed voltage limits, denoted by $\underline{\mathbf{v}}, \overline{\mathbf{v}}$, and respect cable ampacities $\overline{\mathbf{i}}$. Similarly, the apparent power at the substation transformer is denoted by the model $s\left(\mathbf{p}_{t}, \mathbf{q}_{t}, \tilde{s}_{0}\right)$ and should be less than substation transformer rating $\bar{S}$, where $\tilde{s}_{0}$ is the operating complex apparent power used for the power-flow linearization.

The problem consists in maximizing the installed capacity of PV generation while subject to grid constraints. To foster the deployment of the PV plants in nodes with the highest irradiance availability, the installed capacity 
is weighted by the local capacity factor $\gamma_{n}(7)$. The problem formulation is:

$$
\underset{\left\{P_{n}^{\mathrm{pv}} \in \mathbf{R}^{+}, n \in \mathcal{N}\right\}}{\operatorname{maximize}}\left\{\sum_{n \in \mathcal{N}} \gamma_{n} P_{n}^{\mathrm{pv}}\right\}
$$

subject to nodal injections model and grid constraints

$$
\begin{array}{lc}
\mathbf{p}_{t}=\mathbf{p}_{t}^{\mathrm{pv}}-\mathbf{p}_{t}^{\text {load }} & t \in \mathcal{T} \\
\mathbf{q}_{t}=\mathbf{p}_{t}^{\mathrm{pv}}-\mathbf{q}_{t}^{\text {load }} & t \in \mathcal{T} \\
\underline{\mathbf{v}} \leq v\left(\tilde{\mathbf{v}}_{0}, \mathbf{p}_{t}, \mathbf{q}_{t}\right) \leq \overline{\mathbf{v}} & t \in \mathcal{T} \\
\mathbf{0} \leq c\left(\tilde{\mathbf{v}}_{0}, \mathbf{p}_{t}, \mathbf{q}_{t}\right) \leq \overline{\mathbf{i}} & t \in \mathcal{T}, \\
0 \leq s\left(\mathbf{p}_{t}, \mathbf{q}_{t}, \tilde{s}_{0}\right) \leq \bar{S} & t \in \mathcal{T},
\end{array}
$$

and PV generation model and land-availability constraint $\overline{P_{n}^{\mathrm{pv}}}$ :

$$
\begin{array}{lr}
p_{n, t}^{\mathrm{pv}}=g\left(t, n, P_{n}^{\mathrm{pv}}\right) & t \in \mathcal{T}, n \in \mathcal{N} \\
P_{n}^{\mathrm{pv}} \leq \overline{P_{n}^{\mathrm{pv}}} & n \in \mathcal{N} .
\end{array}
$$

\subsection{Increasing PV hosting capacity with BESSs}

\subsubsection{Problem formulation}

The objective of this problem is to determine the optimal location of $\mathrm{PV}$ plants to host a target level of total PV generation capacity, that we denote by $P^{\star}$. However, values of $P^{\star}$ above the grid's PV hosting capacity cannot be accommodated because they would lead to violations of grid constraints. For this reason, this problem also determines an optimal configuration of BESSs (location, converter power ratings, and energy capacities) to relieve grid constraints and enabling the further integration of PV generation in the grid. The results of this process are discussed at the end of this section.

It is worth highlighting that, even if we consider BESSs, the formulation can be extended to other forms of energy storage systems or other resources

\footnotetext{
${ }^{7}$ We include the capacity factor because, even if derived from satellite estimations with coarser resolution that the grid nodes, the topographical shading is at a higher resolution and could impact on the suitability of certain nodes.
} 
capable of providing grid support, like flexible demand [61, 62]. It is also worth highlighting the parallel with PV self-consumption strategies, which can indirectly mitigate the impact of excess PV generation on grid constraints thanks to promoting the direct consumption of locally generated electricity, see e.g. [63, 64]. PV self-consumption is typically provided on a best-effort basis by end consumers and is typically unaware of global grid conditions, thus without offering reliable performance guarantees. Compared to PV selfconsumption, we provide robust guarantees on grid control performance and optimized energy storage requirements considering the whole grid and not a single consumer.

BESSs model. BESSs' active power is denoted by $p_{n, t}^{\text {bess }}$, and reactive by $q_{n, t}^{\text {bess }}$. We model the evolution of the BESS state-of-energy (SOE) with

$$
\mathrm{SOE}_{n, t}=\mathrm{SOE}_{n, t-1}-p_{n, t}^{\text {bess }} \Delta t
$$

where $\Delta t$ is the sampling time. Charging and discharging efficiency is accounted for by integrating the BESS equivalent resistance in the load flow problem as proposed in [65]. If load flow equations are linearized, this modeling choice retains the convexity of the problem without requiring the use of additional variables as, for example, in [66]. Since battery sizes are the decision variables, the optimization problem is solved multiple times taking account of the updated equivalent resistances in proportion to their converter ratings. To implement a safety margin from zero-SOE and full charge, we implement the following constraint

$$
a E_{n}^{\text {bess }} \leq \mathrm{SOE}_{n, t} \leq(1-a) E_{n}^{\text {bess }}
$$

where $0 \leq a \leq 0.5$ is a design parameter and $E_{n}^{\text {bess }}$ is the BESS energy capacity. BESS injections should respect the capability curve of its four quadrant power converter. This reads as:

$$
0 \leq\left(p_{n, t}^{\text {bess }}\right)^{2}+\left(q_{n, t}^{\text {bess }}\right)^{2} \leq\left(P_{n}^{\text {bess }}\right)^{2} .
$$

Capital investment for BESSs and PV plants. The capital investment for installing a PV plant with generation capacity $P^{\mathrm{pv}}$, and a BESS with energy capacity $E_{n}^{b}$ and power rating $P_{n}^{b}$ at node $n$ is: 


$$
J\left(P_{n}^{\mathrm{pv}}, P_{n}^{\mathrm{bess}}, E_{n}^{\mathrm{bess}}\right)=\mathcal{C}_{n}^{\mathrm{pv}} P_{n}^{\mathrm{pv}}+\mathcal{C}^{P} P_{n}^{\text {bess }}+\mathcal{C}^{E} E_{n}^{\mathrm{bess}},
$$

where $\mathcal{C}^{\mathrm{pv}}, \mathcal{C}^{\mathrm{P}}$, and $\mathcal{C}^{\mathrm{E}}$ are the unitary costs for $\mathrm{PV}$, power converter rating, and energy capacity, respectively. Costs are reported in Table 8. They are derived from current market figures.

Table 8: Costs of PV and BESSs.

\begin{tabular}{|c|c|c|}
\hline Component & Unit & Value \\
\hline Turn-key PV system $\left(\mathcal{C}^{\text {pv }}\right)$ & $\mathrm{USD}(\$) / \mathrm{kWp}$ & 1020 \\
BESS converter rating $\left(\mathcal{C}^{\mathrm{P}}\right)$ & $\mathrm{USD}(\$) / \mathrm{kVA}$ & 200 \\
BESS energy capacity $\left(\mathcal{C}^{\mathrm{E}}\right)$ & $\mathrm{USD}(\$) / \mathrm{kWh}$ & 300 \\
\hline
\end{tabular}

Formulation of the decision problem. The decision variables of the problem are the installed PV capacity, the BESS power rating and the BESS energy capacity at all the nodes of the grid, which we collect in the set $\chi=\left\{P_{n}^{\text {pv }}, P_{n}^{\text {bess }}, E_{n}^{\text {bess }} \in \mathbf{R}^{+}, \forall n \in \mathcal{N}\right\}$. Without losing generality, nodes that cannot host PV generation or BESS can be excluded by properly subsetting the nodes index. The problem consists in locating and sizing BESS to accommodate a target level $P^{\star}$ of installed PV generation capacity while minimizing the total capital investment 10 for all the nodes of the grid. The BESSs' optimal location is determined by the battery nodal injections that are different than zeros at certain nodes. Similarly to before, to favour the locations with large PV capacity factors, we weight the installed PV capacity at each node with the factor $\bar{\gamma} / \gamma_{n}$, where $\bar{\gamma}$ is the average among all the capacity factors $\gamma_{n}, n \in \mathcal{N}$ in the network. Finally, the problem is:

$$
\underset{\chi}{\operatorname{minimize}}\left\{\sum_{n \in \mathcal{N}} J\left(\bar{\gamma} / \gamma_{n} \cdot P_{n}^{\mathrm{pv}}, P_{n}^{\mathrm{bess}}, E_{n}^{\mathrm{bess}}\right)\right\}
$$

subject to nodal injections (now with BESSs demand too) and grid constraints

$$
\begin{array}{ll}
\mathbf{p}_{t}=\mathbf{p}_{t}^{\text {pv }}-\mathbf{p}_{t}^{\text {load }}-\mathbf{p}_{t}^{\text {bess }} & t \in \mathcal{T} \\
\mathbf{q}_{t}=\mathbf{p}_{t}^{\text {pv }}-\mathbf{q}_{t}^{\text {load }}-\mathbf{q}_{t}^{\text {bess }} & t \in \mathcal{T} \\
\text { (6d) }-6 \mathrm{ff}, &
\end{array}
$$


BESS model and constraints

$$
\begin{array}{ll}
\mathrm{SOE}_{n, t}=\mathrm{SOE}_{n, t-1}-p_{n, t}^{\text {bess }} \Delta t & t \in \mathcal{T}, n \in \mathcal{N} \\
0 \leq\left(p_{n, t}^{\text {bess }}\right)^{2}+\left(q_{n, t}^{\text {bess }}\right)^{2} \leq\left(P_{n}^{\text {bess }}\right)^{2} & t \in \mathcal{T}, n \in \mathcal{N} \\
a E_{n}^{\text {bess }} \leq \operatorname{SOE}_{n, t} \leq(1-a) E_{n}^{\text {bess }} & t \in \mathcal{T}, n \in \mathcal{N}
\end{array}
$$

and PV model and target PV capacity $P^{\star}$ to install in the grid:

$$
\begin{aligned}
& \sum_{n \in \mathcal{N}} P_{n}^{\mathrm{pv}}=P^{\star} . \\
& \text {.6h }
\end{aligned}
$$

\subsubsection{Results}

For each estimated MV grid, first, we solve the PV problem (6) to obtain the PV hosting capacity, then, we solve the BESS sizing problem (11) by varying $P^{\star}$ in (11i) from $25 \%$ to $300 \%$ (with increments of $25 \%$ ) of the grid PV hosting capacity. It should be noted that both the problems (6) and (11) are solved multiple times for correcting the grid linearization (by updating the injections of newly sized PV and battery installations) and updating battery equivalent resistances (for the battery loss model as previously mentioned). With this procedure, we determine the BESSs requirements for PV configurations below (25-100\%) and above (125-300\%) the grid hosting capacity. The results of this process for are shown in Fig. 14 and are now discussed. Figures 14a and 14b show the cost curves for 10 randomly chosen distribution networks, whereas Figures $14 \mathrm{c}$ and $14 \mathrm{~d}$ show the distribution along the grids with symmetric quantiles. Figure 14a shows the total investment for PV systems and BESSs as a function of the installed PV generation capacity. We can observe two elements. Networks reach a different level of maximum PV installed capacity. This is due to the different values of land availability. Second, the total investment grows at two different rates because the investment, below the hosting capacity, is given by PV panels only, whereas above it, by BESSs too. Figure 14b shows the marginal cost of increasing the level of installed PV generation capacity. We define the marginal cost of each grid as the total cost of the PV-BESS system over the total PV yearly production accounting for the capacity factor as:

$$
\text { Marginal cost }=\sum_{n \in \mathcal{N}} \frac{J\left(\widehat{P}_{n}^{\mathrm{pv}}, \widehat{P}_{n}^{\text {bess }}, \widehat{E}_{n}^{\text {bess }}\right)}{\widehat{P}_{n}^{\mathrm{pv}} \cdot 365 \cdot 24 \cdot \gamma_{n}},
$$


where $\widehat{P}_{n}^{\mathrm{pv}}, \widehat{P}_{n}^{\text {bess }}, \widehat{E}_{n}^{\mathrm{bess}}$ denote the solution of problem $(11)$. It can be seen from Fig. $14 \mathrm{a}$ and $14 \mathrm{~b}$ that, below the hosting capacity, the marginal cost is constant because it corresponds to the unitary cost of PV, whereas above, it increases because progressively larger BESSs are required. Figure 14c and $14 \mathrm{~d}$ shows the density plot of the cost curves derived for all estimated MV networks in Switzerland. They show the distribution of the total and marginal costs among different networks. As it can be seen in Fig. 14a and $14 \mathrm{~b}$, different networks have different PV hosting capacities, therefore the marginal costs of the various systems have different patterns.

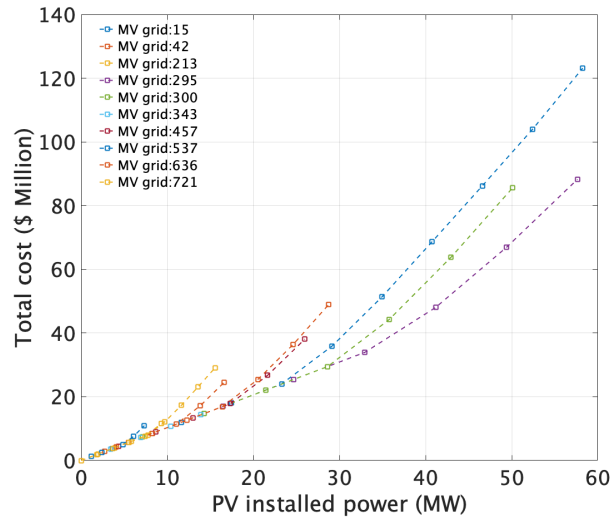

(a)

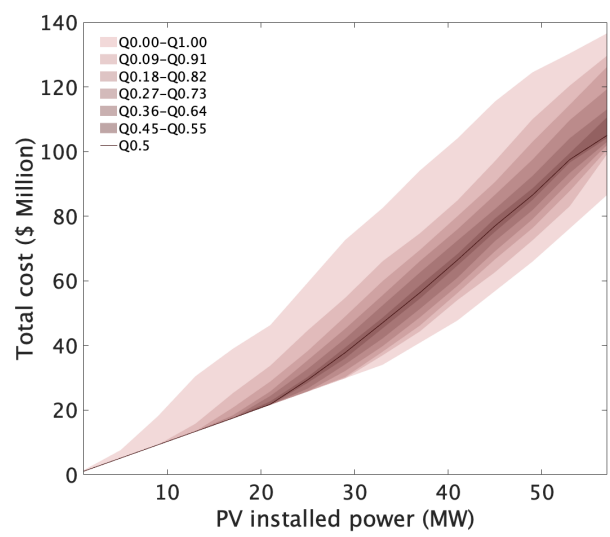

(c)

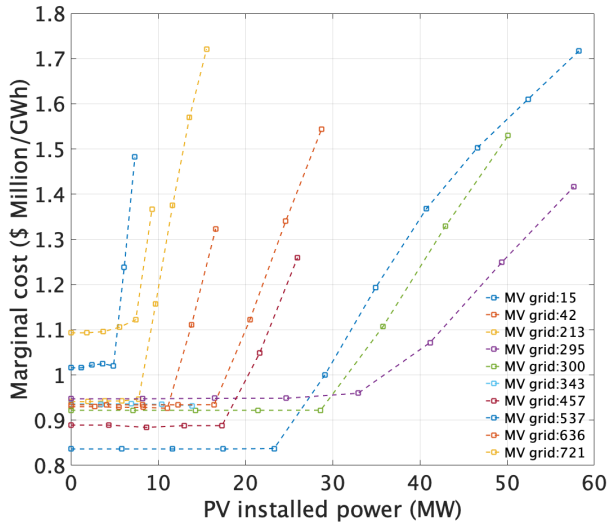

(b)

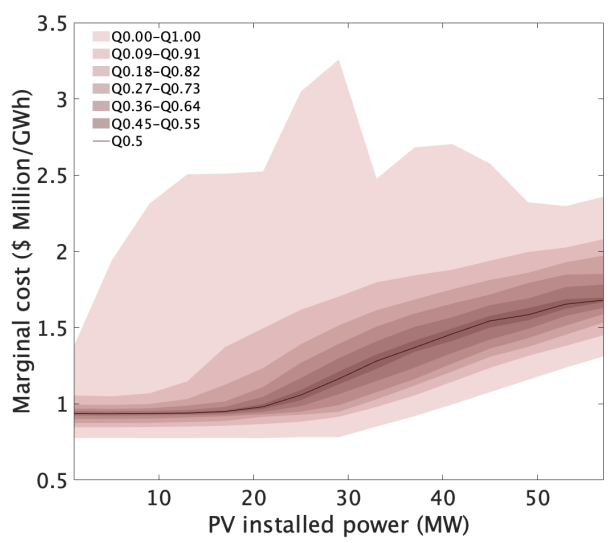

(d)

Figure 14: Investments to achieve a target level of installed PV generation capacity: (a, c) total cost, and (b, d) marginal cost. Top: for randomly chosen 10 MV networks, bottom: for all estimated MV networks in Switzerland (distribution with symmetric quantiles). 


\subsection{Optimal allocation of $P V$ and BESSs}

In the former subsection, we have discussed a method to determine the optimal deployment of PV installations and BESSs within a network to accommodate a target level of installed PV capacity. We have applied it to all identified grids of Section 2 and derived, for each of them, marginal costs for installing increasing levels of installed PV capacity. The estimated marginal costs are key results as they allow us to compare the costs of installing PV generation in various networks across the country, and they will be the fundamental input of the problem discussed in this section. The objective of this problem is to determine the installed PV capacity in each network in order to achieve a countrywide objective for total PV generation at the lowest capital cost.

We denote the curves of Fig. $14 \mathrm{~b}$ with the function $f_{m}\left(P_{m}^{\star}\right)$, where $m \in$ $\mathcal{M}=\{1, \ldots, M\}$ is the index for the identified MV networks and $P_{m}^{\star}$ is the installed capacity in grid $m$. We approximate the curves $f_{m}$ with a piece-wise linear function. The domain of $f_{m}$ is $\left[\underline{P}_{m}^{\star}, \bar{P}_{m}^{\star}\right]$, derived from Fig. $14 \mathrm{~b}$. The problem consists in finding the variables $P_{1}^{\star}, \ldots, P_{M}^{\star}$ at the minimum total cost and such that the total installed capacity equals the countrywide PV installation target $P^{\text {target }}$. The problem is:

$$
\underset{\left\{P_{m}^{\star} \in \mathbf{R}^{+}, m \in \mathcal{M}\right\}}{\operatorname{minimize}}\left\{\sum_{m \in \mathcal{M}} \mathcal{P}_{m}^{\mathrm{pv}} f_{m}\left(\mathcal{P}_{m}^{\mathrm{pv}}\right)\right\}
$$

subject to the domains of the variables and the PV installation target:

$$
\begin{array}{ll}
\underline{P}_{m}^{\star} \leq P_{m}^{\star} \leq \bar{P}_{m}^{\star} & m \in \mathcal{M} \\
\sum_{m \in \mathcal{M}} P_{m}^{\star}=P^{\text {target }} . &
\end{array}
$$

The results are discussed in the next section.

\section{Results and Discussion}

\subsection{Case study}

In the previous sections, we have presented a modeling toolchain that determines an economically optimal deployment of PV plants and BESSs to achieve a target level of installed PV generation while accounting for the 
capacity factor spatial distribution, grids constraints and how they can be relieved by BESSs when the PV generation capacity exceeds the grid's PV hosting capacity. It is worth highlighting that the problem's essence is not only about achieving an optimal deployment of PV generation based on its countrywide potential but also extending with distributed energy storage the PV hosting capacity of grids with large PV generation potential if this leads to more economically convenient configurations. For example, as shown in this section, it is more convenient to invest in BESSs to extend the hosting capacity of a grid with a large generation potential and installing here additional PV generation rather than in grids with lower generation potential.

In this section, we compare this approach (that we call Case 1) against the case where the same level of installed PV generation capacity is deployed uniformly in the distribution grids (Case 0). For an illustrative comparison between Case 0 and 1, we refer to Fig. $14 \mathrm{~d}$; for a given value of total PV generation capacity, Case 0 involves selecting, for each network, an installed PV generation capacity ( $\mathrm{x}$-axis) that is proportional to the grid area and regardless of its cost (y-axis). Case 1 involves placing PV generation starting from the grid with the lowest cost (y-axis), and saturating its potential (sweeping the $\mathrm{x}$-axis) before moving to the second cheapest grid.

\subsection{Deployment of $P V$ plants}

Figure 15 shows the distribution of installed PV generation capacity across Switzerland for increasing (from top to bottom) levels of total installed capacity and for Case 0 (left column) and Case 1 (right). The difference between the two deployment policies is evident by comparing the plots in the first row: in the left plot (Case 0), PV plants are installed uniformly in the grids ${ }^{8}$, whereas in the right plot (Case 1) PV is installed prioritizing regions with higher irradiance availability, which appear to be Ticino, Leman and Neuchatel regions, and west Valais.

For increasing values of installed PV capacity (second and third rows of Fig. 15), it can be observed that Case 0 and Case 1 feature increasingly similar geographical distribution patterns. This is due to land-use limitations, and the activation of the associated constraint in (6h). In other words, once Case 1 saturates the available locations for PV deployment in regions with

\footnotetext{
${ }^{8}$ Non-uniform spatial distribution over the country of PV generation is because grids are not uniformly distributed.
} 


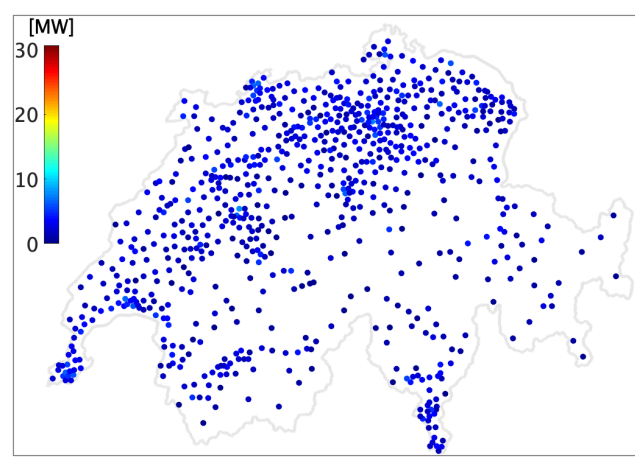

(a)

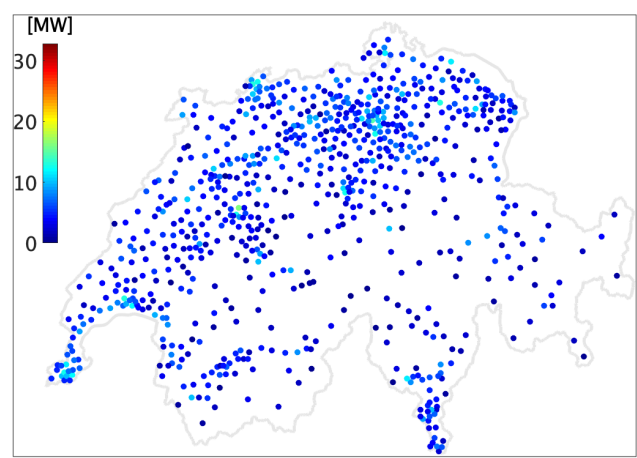

(c)

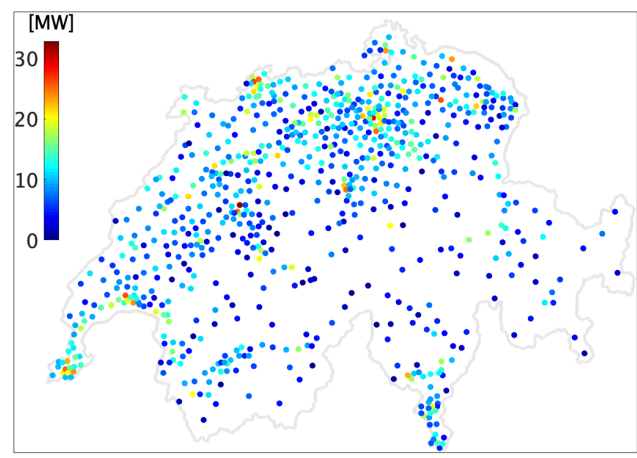

(e)

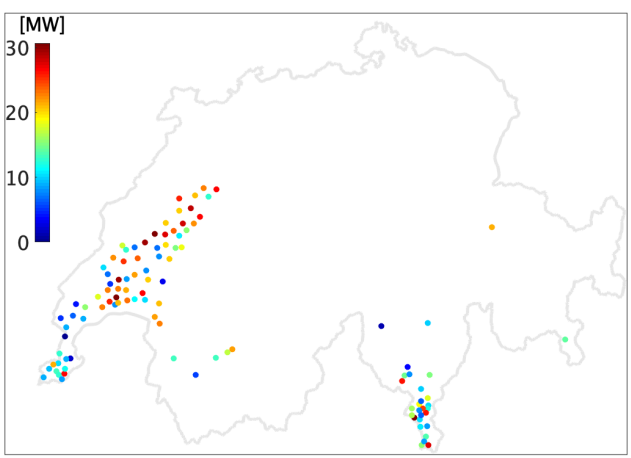

(b)

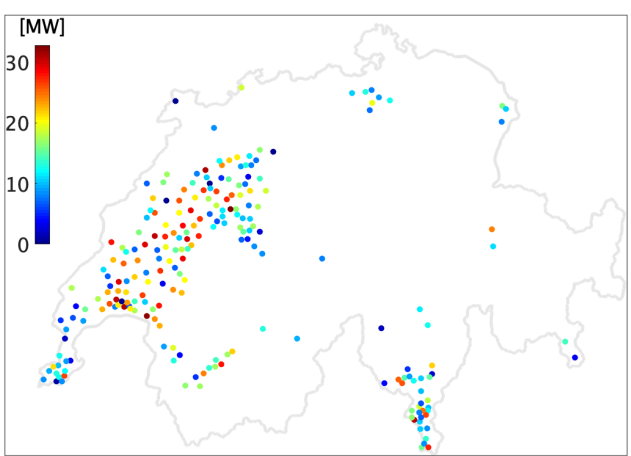

(d)

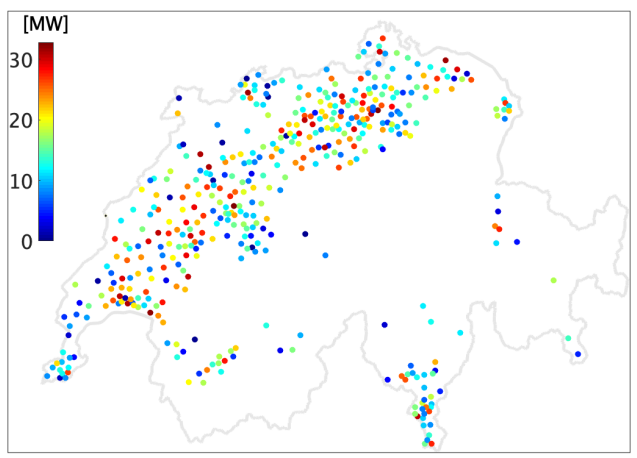

(f)

Figure 15: Installed PV generation capacity (in MW) across Switzerland for scenario A in (a) and (b), scenario B in (c) and (d), and scenario C in (e) and (f), for Case 0 and Case 1, respectively. Scenarios refer to the installed capacity of PV generation and are defined in Table 9. 
high irradiance potential, it starts installing PV generation in second-choice grids. The distribution of the BESS follows the same pattern as of PV.

\subsection{Deployment of PV plants and BESSs for Case 0 and Case 1}

Table 9 shows the PV installed capacity, the yearly production, the BESS power rating and energy capacity, and the total cost (i.e., investments for PV plants and BESSs) for 10 scenarios (A-J) of PV generation deployment for Case 0 and Case 1. Scenarios A, B, C to J correspond to allocating PV generation in 5, 10, 20 to $90 \%$ (with increments of 10\%), respectively, of the available surface. We remind that the available surface for PV is $10 \%$ of the settlement areas, as discussed in section 3.1.2. The energy transition scenario for Switzerland reported in [67] estimates a yearly PV production potential from roof-top PV around $25 \mathrm{TWh}$, that corresponds to our scenarios H-J.

From Table 9 we can make the following observations.

- Case 1/Scenario A achieves a 0.21 TWh increase in yearly production compared to the same scenario of Case 0 thanks to installing PV generation in distribution grids with larger PV generation potential first. For increasing values of installed capacity (scenarios from B to J), the yearly production of the two cases converges to the same values due to land-use limitations, as discussed in 4.2 ;

- Case 0 requires BESSs starting from Scenario C, whereas Case 1 has mild needs in Scenario B already. This denotes that it is more cost effective to invest in BESS to increase the hosting capacity of high PV-generation-potential grids rather than connecting that same PV capacity in other grids with less PV generation potential.

- Connecting PV generation above Scenario $\mathrm{C}$ in Case 0 requires progressively larger values of energy storage capacity and power rating. For example, doubling its installed capacity (from 6.85 to $13.70 \mathrm{GW}$ ) requires nearly 40 times the energy storage capacity (from 0.14 to 5.73 GWh). It is worth noting that the needs for BESSs increases sharper for Case 0 than Case 1. This is because the latter problem optimizes the locations of BESSs and PV across all the grids attaining a minimum costs, whereas Case 0 scales PV capacity regardless of grid properties and irradiance potential. Costs are discussed next. 
Table 9: Deployment of PV and BESS in the two cases.

\begin{tabular}{|c|c|c|c|c|c|c|c|c|c|}
\hline \multirow[t]{2}{*}{ Scenario } & \multirow{2}{*}{$\begin{array}{c}\text { PV installed } \\
\text { capacity } \\
(\text { GWp) }\end{array}$} & \multicolumn{2}{|c|}{$\begin{array}{c}\text { PV production } \\
(\mathrm{TWh} / \mathrm{y})\end{array}$} & \multicolumn{2}{|c|}{$\begin{array}{l}\text { BESS Power } \\
(\text { GW })\end{array}$} & \multicolumn{2}{|c|}{$\begin{array}{c}\text { BESS Capacity } \\
(\text { GWh })\end{array}$} & \multicolumn{2}{|c|}{$\begin{array}{l}\text { Total cost } \\
\text { Billions } \$\end{array}$} \\
\hline & & Case 0 & Case 1 & Case 0 & Case 1 & Case 0 & Case 1 & Case 0 & Case 1 \\
\hline A & 1.71 & 1.90 & 2.11 & 0.00 & 0.00 & 0.00 & 0.00 & 1.76 & 1.77 \\
\hline B & 3.43 & 3.81 & 4.09 & 0.00 & 0.00 & 0.00 & 0.01 & 3.53 & 3.53 \\
\hline $\mathrm{C}$ & 6.85 & 7.62 & 7.87 & 0.03 & 0.01 & 0.14 & 0.01 & 7.11 & 7.07 \\
\hline $\mathrm{D}$ & 10.28 & 11.42 & 11.46 & 0.46 & 0.04 & 1.31 & 0.05 & 11.07 & 10.61 \\
\hline $\mathrm{E}$ & 13.70 & 15.23 & 15.25 & 1.74 & 0.51 & 5.73 & 0.68 & 16.18 & 14.42 \\
\hline $\mathrm{F}$ & 17.02 & 18.92 & 18.99 & 3.64 & 3.08 & 14.74 & 7.88 & 22.68 & 20.51 \\
\hline G & 20.11 & 22.36 & 22.43 & 5.87 & 5.67 & 26.83 & 21.26 & 29.94 & 28.23 \\
\hline $\mathrm{H}$ & 22.89 & 25.44 & 25.46 & 8.10 & 7.94 & 39.90 & 36.20 & 37.17 & 36.03 \\
\hline I & 25.42 & 28.24 & 28.25 & 10.18 & 10.08 & 53.41 & 51.00 & 44.25 & 43.50 \\
\hline $\mathrm{J}$ & 27.57 & 30.61 & 30.61 & 12.07 & 12.00 & 65.87 & 65.04 & 50.57 & 50.31 \\
\hline
\end{tabular}

\subsection{Cost comparison}

Figure 16 compares the marginal cost (i.e., total cost divided by the PV yearly production for the respective scenarios) of the two cases using results from Table 9. Case 1 (optimal allocation) always achieves a lower unitary

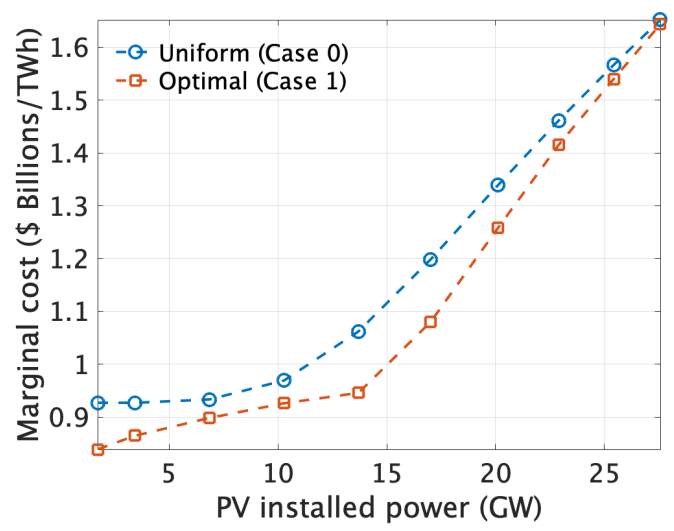

Figure 16: Cost per TWh of PV energy production for the two cases.

cost compared to Case 0. This is because the optimal allocation problem places the PV plants at locations with the higher irradiance potential first, whereas Case 0 (uniform PV allocation) places the PV plants proportionally to the available area. This shows the effectiveness of the optimal allocation algorithm. However, for higher values of installed PV generation capacity, 
the costs converge to the same value due to land-use limitations in most PV-favourable grids.

Fig. 17a shows the BESS energy capacity and power rating requirements for the optimal case as a function of the installed PV generation capacity using the results from Table 9. The energy storage requirements are mild, before increasing sharply after $14 \mathrm{GW}\left({ }^{9}\right)$. It can be noted that mitigating with BESSs the impact of excess PV generation on distribution grids is an energy-intensive application, with power-rating-to-energy-capacity ratios (i.e., C-rates) around 1/5. As current BESSs technologies can safely operate up to $2-3 \mathrm{C}$, the spare power rating can be conveniently used to provide additional ancillary services, such as primary frequency control and grid synchronization services, that are mostly power-intensive [68]. Fig. 17b shows the corresponding system cost and cost breakdown and shows that the cost of the PV panels is largely dominant.

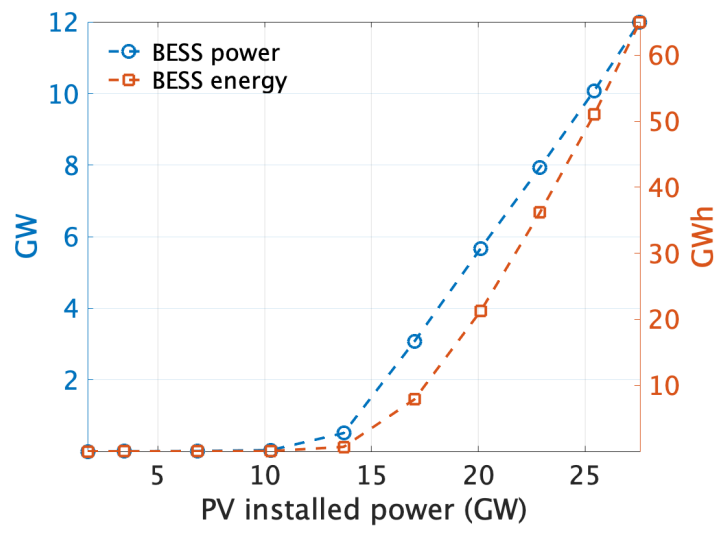

(a)

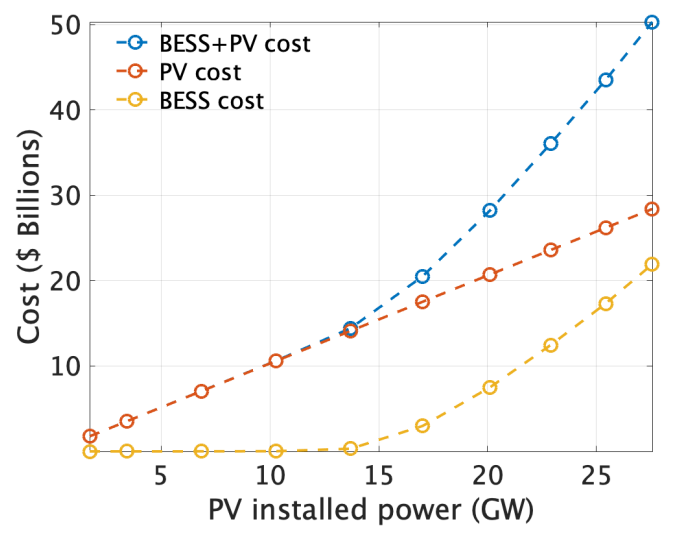

(b)

Figure 17: (a) BESS cost and size: (a) BESSs power rating and energy capacity and (b) system cost breakdown for Case 1 for different levels of installed PV generation capacity.

\footnotetext{
${ }^{9}$ This value of hosting capacity is in-line with the countrywide hosting capacity obtained by solving the problem in Section 3.2 .
} 


\section{Conclusions}

PV generation will be key in achieving the energy transition targets, in Switzerland and other countries. As PV plants are connected to the power distribution system, it is important to consider the generation hosting capacity of existing distribution grids, which is typically limited due to grid operators' requirements to keep voltage levels within statutory limits, respect the cable ampacities and rating of the substation transformer.

The main obstacle to analyzing the PV hosting capacity of existing distribution grids is that their topology and line characteristics are confidential information owned by different DSOs. For this reason, we have first developed a method to estimate likely distribution grids starting from publicly available georeferenced data. Relying on the fact that existing distribution grids interface electrical demand, we use the countrywide geographical distribution of the electrical demand to infer the HV and MV electrical nodes' locations and connect them with a routing procedure from the existing literature. We then present a computationally tractable method based on a linearized OPF problem to compute the PV hosting capacity of distribution grids, including how to host PV generation beyond prescribed limits with adequately located and sized distributed energy storage systems for relieving grid constraints violations.

Finally, we propose a specific planning problem that determines a costefficient allocation of PV power across the whole country, accounting for the technical limitations of the distribution grids (including adding energy storage, if conducive to lower system costs) and the distributed potential of PV generation, modeled with highly resolved PV capacity factors from the PVGIS database. We also consider land-use constraints to identify the sites where it is possible to install PV generation. The "cost-efficiency" notion for installing PV and energy storage systems includes two factors. First, cost efficiency is higher when installing PV plants where their capacity factor is larger. Second, it may be more cost-efficient to invest in distributed energy storage to extend the PV hosting capacity of highly insulated distribution grids rather than installing PV plants where their capacity factor is low.

The impact of this paper is twofold. On the one hand, it provides to distribution system operator a mathematically tractable and interpretable method to assess the PV generation hosting capacity of distribution grids, including how to cost optimally extend it with energy storage systems. On the other hand, developed methods provide actionable indications to national 
policymakers on the level of PV generation that a country can host and, on its techno-economical optimal deployment.

\section{Appendix A. Verification of optimal power flow results}

We compare the voltage and current magnitudes computed by the linearized OPF model of Sec. 3.2 and 3.3 against ground-truth values from an AC load flow. The analysis is done for one of the synthetically generated network for which, the topology (with line parameters) and the nominal injections are shown in Fig. A.18 and Table A.10 respectively. As mentioned in Sec 3.3.2, the OPFs are solved by successively linearizing the model accounting for the updated BESS and PV injections to correct the linearization error until the cost of the problem converges. Fig. A.19a and Fig. A.19b show the power and energy ratings and the respective costs determined by the OPF of Sec. 3.3, respectively. Results settle in 7 iterations. After convergence is reached, we check the accuracy of the linear grid model against non-linear AC power flow using the BESS and PV injections from OPF problem. Fig. A.20 shows the CDF plots of error of the nodal voltage magnitude and currents modeled by the linear OPF and the AC power flow. Table A.11 shows the maximum, absolute mean and mean error for the voltage and current modeling. They show that the voltage and current modeling errors are below $0.5 \%$ and $1.75 \%$ respectively. This proves that the voltage and current constraints modeling using sensitivity-based linear grid model is close to the non-linear $\mathrm{AC}$ power flow.

Table A.10: Nominal Load and PV per node

\begin{tabular}{|l|c|c||l|c|c|}
\hline Node & Load [MW] & PV [MWp] & Node & Load [MW] & PV [MWp] \\
\hline N1 & - & - & N14 & 0.17 & - \\
N2 & - & 1.05 & N15 & 0.18 & 0.35 \\
N3 & 0.22 & - & N16 & 0.19 & - \\
N4 & 0.15 & - & N17 & 0.17 & 0.44 \\
N5 & 0.14 & - & N18 & 0.20 & - \\
N6 & 0.21 & 1.75 & N19 & 0.21 & - \\
N7 & 0.17 & 1.90 & N20 & 0.22 & - \\
N8 & 0.19 & 0.87 & N21 & 0.16 & - \\
N9 & 0.20 & 1.16 & N22 & 0.18 & 1.00 \\
N10 & 0.19 & 0.70 & N23 & 0.26 & 1.81 \\
N11 & 0.14 & - & N24 & 0.23 & 1.17 \\
N12 & 0.17 & - & N25 & 0.02 & - \\
N13 & 0.17 & - & & & \\
\hline
\end{tabular}




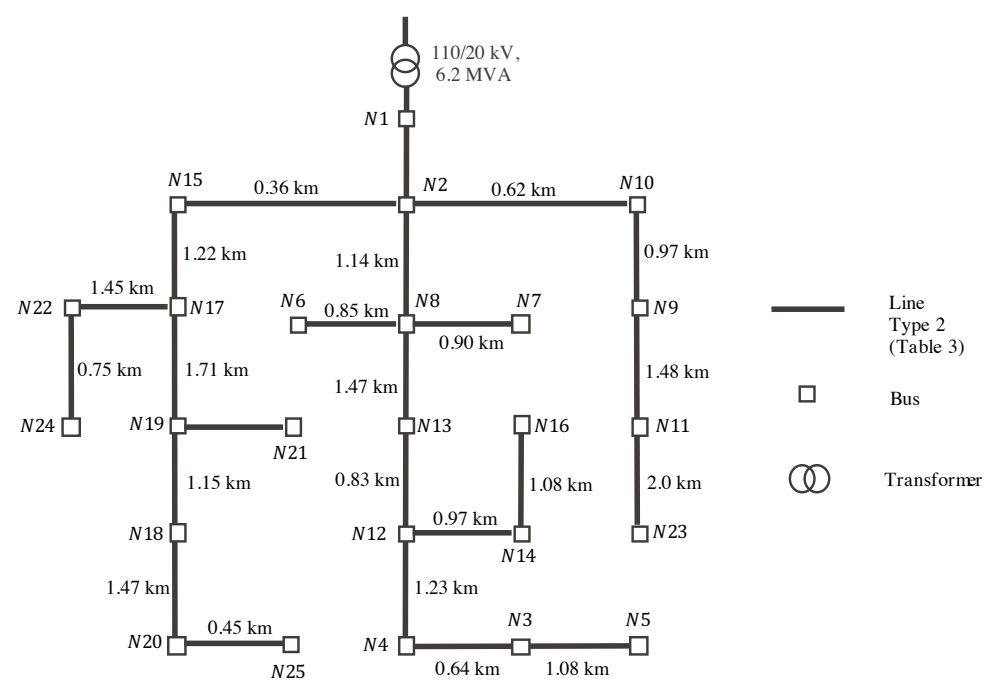

Figure A.18: One of synthetically generated network as test case for the verification of linear grid model.

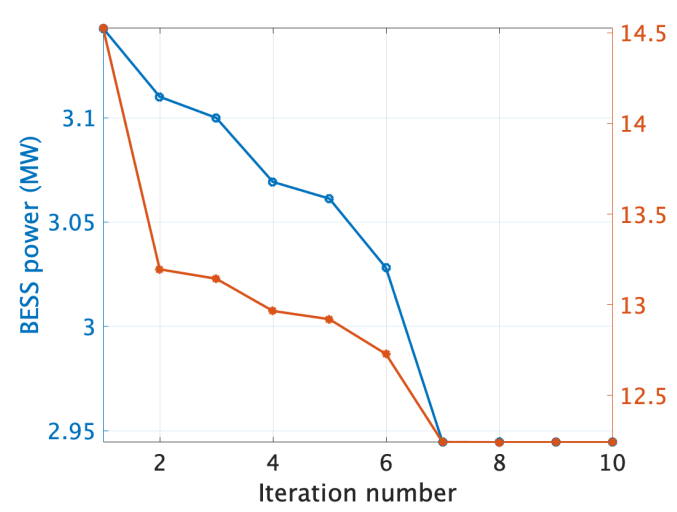

(a)

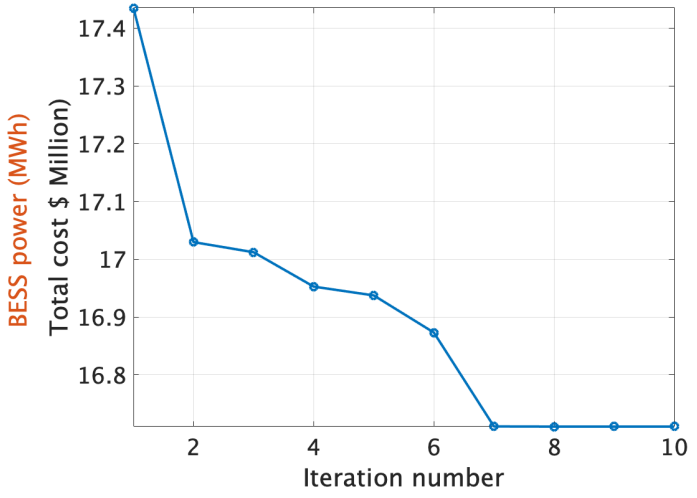

(b)

Figure A.19: Plots showing convergence of the BESS sizes and the objective by correcting the linear power flow coefficients with newest battery injections from previous iteration: (a) BESS power and energy size and (b) Cost of the PV-BESS system.

\section{Appendix B. Validation of synthetically generated MV networks}

We compare two estimated grids from our model with a real distribution network in Aigle, Switzerland, for which it was possible to access the topol- 
Table A.11: Accuracy of the linear power flow.

\begin{tabular}{|l|c|c|c|}
\hline & Max & SD & Mean \\
\hline Nodal voltage error & $4.2 \mathrm{e}-3$ & $1.8 \mathrm{e}-3$ & $1.1 \mathrm{e}-3$ \\
\hline Lines currents error & $1.75 \mathrm{e}-2$ & $4.1 \mathrm{e}-3$ & $4.8 \mathrm{e}-4$ \\
\hline
\end{tabular}

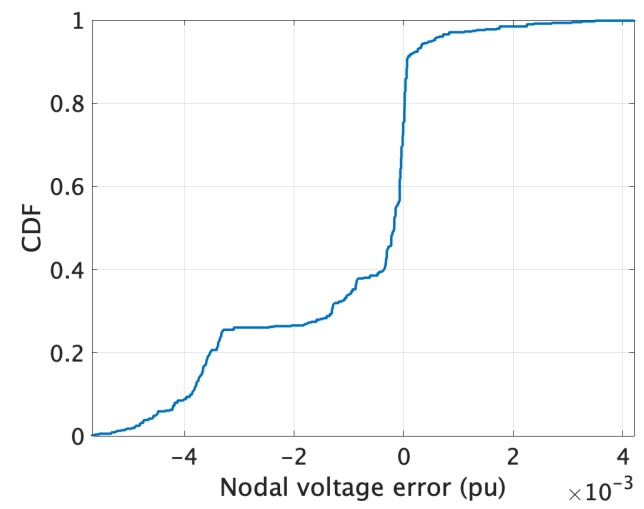

(a)

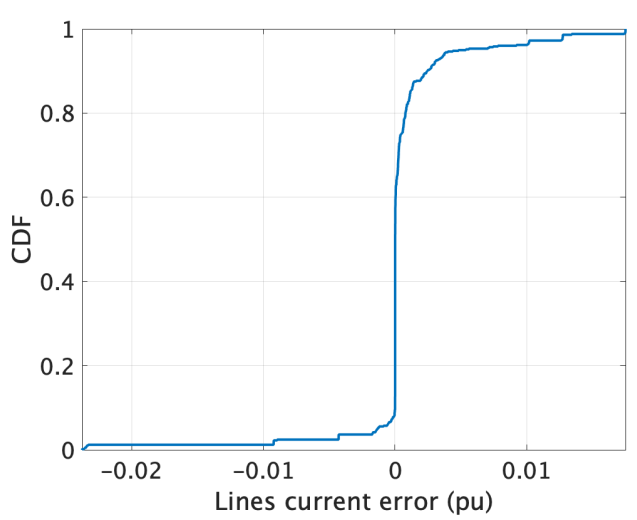

(b)

Figure A.20: CDF plots (a) nodal voltages error and (b) branch current error.

ogy and grid data. It is a three-phase $21 \mathrm{kV} / 6 \mathrm{MVA}$, a 55 -bus network. The two synthetically generated networks are picked from a region near Aigle. The validation refers to comparing the "loadability" of the network, namely evaluating the CDFs of the voltage and line current magnitudes at different load conditions. For the comparison, we use the load profiles shown in Fig. 13a. Fig. B.21 shows the CDFs of the voltage and current magnitudes of the original and estimated networks. The maximum, mean and the minimum values are reported in the Table B.12. The numerical comparison and the CDFs show a good match among the networks. In particular, it emerges that the voltage and current magnitudes of the estimated networks fall in the same ranges as the one of the real grid. 


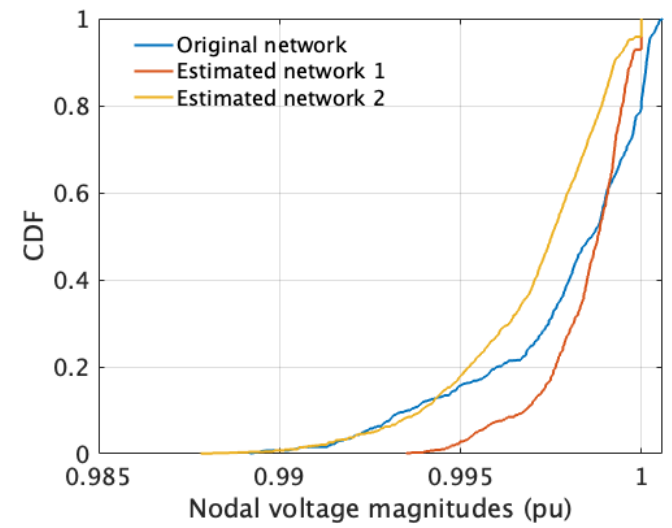

(a)

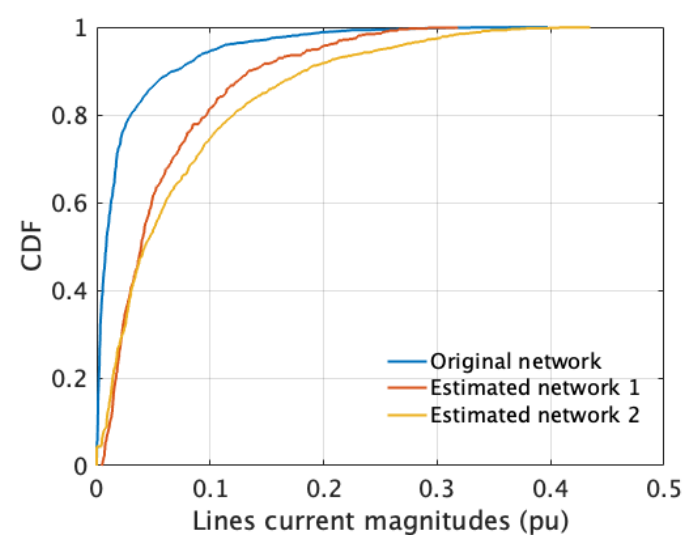

(b)

Figure B.21: CDF plots: (a) nodal voltages and (b) lines currents of original network and the estimated networks shown in different colors.

Table B.12: Comparison of actual and estimated networks.

\begin{tabular}{|l|c|c|c|c|c|c|}
\hline Networks & \multicolumn{3}{|c|}{ Nodal voltage magnitudes } & \multicolumn{3}{c|}{ Lines current magnitudes } \\
\hline & Max & SD & Mean & Max & SD & Mean \\
\hline Actual & 1.0006 & 0.0025 & 0.9978 & 0.3974 & 0.0420 & 0.0237 \\
\hline Estimated case 1 & 1.0000 & 0.0013 & 0.9985 & 0.3181 & 0.0570 & 0.0593 \\
\hline Estimated case 2 & 1.0000 & 0.0022 & 0.9970 & 0.4346 & 0.0782 & 0.0732 \\
\hline
\end{tabular}

\section{References}

[1] International Renewable Energy Agency, Future of Solar Photovoltaic: Deployment, investment, technology, grid integration and socio-economic aspects, Technical Report, 2019.

[2] A. Jäger-Waldau, Pv status report 2019, eur 29938 en, Publications Office of the European Union, Luxembourg (2019).

[3] International Energy Agency, PVPS T7-4, Potential for building integrated photovoltaics, Technical Report, 2002.

[4] C. P. Castillo, F. B. e Silva, C. Lavalle, An assessment of the regional potential for solar power generation in eu-28, Energy policy 88 (2016) 86-99. 
[5] L. Wiginton, H. T. Nguyen, J. M. Pearce, Quantifying rooftop solar photovoltaic potential for regional renewable energy policy, Computers, Environment and Urban Systems 34 (2010) 345-357.

[6] K. Orehounig, G. Mavromatidis, R. Evins, V. Dorer, J. Carmeliet, Towards an energy sustainable community: An energy system analysis for a village in switzerland, Energy and Buildings 84 (2014) 277-286.

[7] G. Mavromatidis, K. Orehounig, J. Carmeliet, Evaluation of photovoltaic integration potential in a village, Solar Energy 121 (2015) 152-168.

[8] R. Singh, R. Banerjee, Estimation of rooftop solar photovoltaic potential of a city, Solar Energy 115 (2015) 589-602.

[9] N. Mohajeri, G. Upadhyay, A. Gudmundsson, D. Assouline, J. Kampf, J.-L. Scartezzini, Effects of urban compactness on solar energy potential, Renewable Energy 93 (2016) 469-482.

[10] N. Mohajeri, A. Gudmundsson, T. Kunckler, G. Upadhyay, D. Assouline, J. Kampf, J. Scartezzini, A solar-based sustainable urban design: The effects of city-scale streetcanyon geometry on solar access in geneva, switzerland, Applied Energy 240 (2019) $173-190$.

[11] K. Bódis, I. Kougias, A. Jäger-Waldau, N. Taylor, S. Szabó, A high-resolution geospatial assessment of the rooftop solar photovoltaic potential in the European Union, Renewable and Sustainable Energy Reviews 114 (2019) 109309.

[12] R. Cossent, L. Olmos, T. Gómez, C. Mateo, P. Frías, Distribution network costs under different penetration levels of distributed generation, European Transactions on Electrical Power 21 (2011) 1869-1888.

[13] A. Navarro, L. F. Ochoa, D. Randles, Monte carlo-based assessment of pv impacts on real uk low voltage networks, in: 2013 IEEE Power Energy Society General Meeting, 2013, pp. 1-5.

[14] A. Navarro, L. F. Ochoa, D. Randles, Monte carlo-based assessment of pv impacts on real uk low voltage networks, in: 2013 IEEE Power \& Energy Society General Meeting, IEEE, 2013, pp. 1-5.

[15] A. Arshad, M. Lindner, M. Lehtonen, An analysis of photo-voltaic hosting capacity in finnish low voltage distribution networks, Energies 10 (2017) 1702.

[16] F. Ebe, B. Idlbi, J. Morris, G. Heilscher, F. Meier, Evaluation of pv hosting capacity of distribuion grids considering a solar roof potential analysis - comparison of different algorithms, in: 2017 IEEE Manchester PowerTech, IEEE, 2017, pp. 1-6. 
[17] H. Al-Saadi, R. Zivanovic, S. F. Al-Sarawi, Probabilistic hosting capacity for active distribution networks, IEEE Transactions on Industrial Informatics 13 (2017) 25192532 .

[18] F. Sossan, J. Darulova, M. Paolone, A. Kahl, S. J. Bartlett, M. Lehning, Large scale deployment of pv units in existing distribution networks: Optimization of the installation layout, in: 2016 Power Systems Computation Conference (PSCC), IEEE, 2016, pp. 1-6.

[19] A. Fuchs, T. Demiray, E. Panos, R. Kannan, T. Kober, C. Bauer, W. Schenler, P. Burgherr, S. Hirschberg, ISCHESS-Integration of stochastic renewables in the Swiss electricity supply system, Technical Report, ETH Zurich, 2017.

[20] Z. Wang, A. Scaglione, R. J. Thomas, Generating statistically correct random topologies for testing smart grid communication and control networks, IEEE transactions on Smart Grid 1 (2010) 28-39.

[21] P. Schultz, J. Heitzig, J. Kurths, A random growth model for power grids and other spatially embedded infrastructure networks, The European Physical Journal Special Topics 223 (2014) 2593-2610.

[22] S. Soltan, G. Zussman, Generation of synthetic spatially embedded power grid networks, in: 2016 IEEE Power and Energy Society General Meeting (PESGM), IEEE, 2016, pp. 1-5.

[23] A. B. Birchfield, T. Xu, K. M. Gegner, K. S. Shetye, T. J. Overbye, Grid structural characteristics as validation criteria for synthetic networks, IEEE Transactions on power systems 32 (2016) 3258-3265.

[24] W. Medjroubi, U. P. Müller, M. Scharf, C. Matke, D. Kleinhans, Open data in power grid modelling: new approaches towards transparent grid models, Energy Reports 3 (2017) 14-21.

[25] H. Rui, M. Arnold, W. H. Wellssow, Synthetic medium voltage grids for the assessment of smart grid techniques, in: 2012 3rd IEEE PES Innovative Smart Grid Technologies Europe (ISGT Europe), IEEE, 2012, pp. 1-8.

[26] J. Amme, G. Pleßmann, J. Bühler, L. Hülk, E. Kötter, P. Schwaegerl, The ego grid model: An open-source and open-data based synthetic medium-voltage grid model for distribution power supply systems, in: Journal of Physics: Conference Series, volume 977, 2018.

[27] U. P. Müller, I. Cussmann, C. Wingenbach, J. Wendiggensen, AC power flow simulations within an open data model of a high voltage grid, in: Advances and New Trends in Environmental Informatics, Springer, 2017, pp. 181-193. 
[28] U. P. Müller, L. Wienholt, D. Kleinhans, I. Cußmann, W.-D. Bunke, G. Pleßmann, J. Wendiggensen, The ego grid model: An open source approach towards a model of german high and extra-high voltage power grids, in: Journal of Physics: Conference Series, volume 977, IOP Publishing, 2018, p. 012003.

[29] D. Assouline, N. Mohajeri, J.-L. Scartezzini, Quantifying rooftop photovoltaic solar energy potential: A machine learning approach, Solar Energy 141 (2017) 278-296.

[30] D. Assouline, N. Mohajeri, J.-L. Scartezzini, Large-scale rooftop solar photovoltaic technical potential estimation using random forests, Applied energy 217 (2018) 189211.

[31] A. Walch, R. Castello, N. Mohajeri, J.-L. Scartezzini, Big data mining for the estimation of hourly rooftop photovoltaic potential and its uncertainty, Applied Energy $262(2020)$.

[32] Entso-e transmission system map, 2019. URL: https://www.ent soe.eu/data/ map/.

[33] B. Wiegmans, Gridkit extract of entso-e interactive map, 2016. URL: https:// doi.org/10.5281/zenodo.55853, doi $10.5281 /$ zenodo.55853.

[34] S. Wang, Z. Lu, S. Ge, C. Wang, An improved substation locating and sizing method based on the weighted voronoi diagram and the transportation model, Journal of Applied Mathematics 2014 (2014).

[35] Y. Chen, S. Wang, J. Yu, W. Li, X. Shi, W. Yang, Optimal weighted voronoi diagram method of distribution network planning considering city planning coordination factors, in: 2017 4th International Conference on Systems and Informatics (ICSAI), IEEE, 2017, pp. 335-340.

[36] Y. Yan, F. Wang, K. Zhou, S. Chen, J. Huang, B. Wen, Substation sower supply area division based on voronoi diagram and road grid, in: IOP Conference Series: Materials Science and Engineering, volume 677, IOP Publishing, 2019, p. 042101.

[37] L. Eymann, J. Rohrer, M. Stucki, Energieverbrauch der schweizer kantone: Endenergieverbrauch und mittelabfluss durch den energie-import (2014).

[38] K. Wojdyga, An influence of weather conditions on heat demand in district heating systems, Energy and Buildings 40 (2008) 2009-2014.

[39] S. Avdakovic, A. Ademovic, A. Nuhanovic, Correlation between air temperature and electricity demand by linear regression and wavelet coherence approach: UK, Slovakia and Bosnia and Herzegovina case study, Archives of Electrical Engineering 62 (2013).

[40] R. C. Gonzalez, R. E. Woods, S. L. Eddins, Digital image processing using MATLAB, Pearson Education India, 2004. 
[41] Swiss Federal Office of Energy, Statistique Suisse de L'électricité 2015, Technical Report, 2016.

[42] CIGRE' Task Force C6.04.02, Benchmark Systems for Network Integration of renewable and Distributed Energy Resources, Technical Report, 2009.

[43] P. S. Georgilakis, N. D. Hatziargyriou, A review of power distribution planning in the modern power systems era: Models, methods and future research, Electric Power Systems Research 121 (2015) 89-100.

[44] S. Najafi, S. H. Hosseinian, M. Abedi, A. Vahidnia, S. Abachezadeh, A framework for optimal planning in large distribution networks, IEEE Transactions on Power Systems 24 (2009) 1019-1028.

[45] J. Gomez, H. Khodr, P. De Oliveira, L. Ocque, J. Yusta, R. Villasana, A. Urdaneta, Ant colony system algorithm for the planning of primary distribution circuits, IEEE Transactions on power systems 19 (2004) 996-1004.

[46] E. Diaz-Dorado, J. Cidrás, E. Míguez, Application of evolutionary algorithms for the planning of urban distribution networks of medium voltage, IEEE Transactions on Power Systems 17 (2002) 879-884.

[47] S. Afifi, D.-C. Dang, A. Moukrim, A simulated annealing algorithm for the vehicle routing problem with time windows and synchronization constraints, in: International Conference on Learning and Intelligent Optimization, Springer, 2013, pp. 259-265.

[48] S. Goswami, Distribution system planning using branch exchange technique, IEEE Transactions on Power Systems 12 (1997) 718-723.

[49] G. Peponis, M. Papadopoulos, New dynamic, branch exchange method for optimal distribution system planning, IEE Proceedings-Generation, Transmission and Distribution 144 (1997) 333-339.

[50] N. G. Boulaxis, M. P. Papadopoulos, Optimal feeder routing in distribution system planning using dynamic programming technique and gis facilities, IEEE Transactions on Power Delivery 17 (2002) 242-247.

[51] J. M. Nahman, D. M. Peric, Optimal planning of radial distribution networks by simulated annealing technique, IEEE Transactions on Power Systems 23 (2008) 790795 .

[52] J. M. Nahman, D. M. Perić, Radial distribution network planning under uncertainty by applying different reliability cost models, International Journal of Electrical Power \& Energy Systems 117 (2020) 105655.

[53] F. Pilo, S. Jupe, F. Silvestro, C. Abbey, A. Baitch, B. Bak-Jensen, C. Carter-Brown, G. Celli, K. El, M. F. Bakari, et al., Planning and optimization methods for active distribution systems (2014). 
[54] EN 50160, Voltage characteristics of electricity supplied by public distribution systems, European standard (1999).

[55] M. Nick, R. Cherkaoui, M. Paolone, Optimal planning of distributed energy storage systems in active distribution networks embedding grid reconfiguration, IEEE Transactions on Power Systems 33 (2017) 1577-1590.

[56] JRC Photovoltaic Geographical Information System (PVGIS) - European Commission, 2016. URL: https://re.jrc.ec.europa.eu/pvg_tools/en/tools. html.

[57] A. Walch, N. Mohajeri, J.-L. Scartezzini, A critical comparison of methods to estimate solar rooftop photovoltaic potential in switzerland, in: Journal of Physics: Conference Series, volume 1343, IOP Publishing, 2019, p. 012035.

[58] R. Buffat, S. Grassi, M. Raubal, A scalable method for estimating rooftop solar irradiation potential over large regions, Applied energy 216 (2018) 389-401.

[59] F. Sossan, E. Scolari, R. Gupta, M. Paolone, Solar irradiance estimations for modeling the variability of photovoltaic generation and assessing violations of grid constraints: A comparison between satellite and pyranometers measurements with load flow simulations, Journal of Renewable and Sustainable Energy 11 (2019) 056103.

[60] R. Gupta, F. Sossan, M. Paolone, Performance Assessment of Linearized OPF-based Distributed Real-time Predictive Control, in: IEEE PowerTech 2019, Milan, Italy, 2019.

[61] F. Sossan, Equivalent electricity storage capacity of domestic thermostatically controlled loads, Energy 122 (2017) 767-778.

[62] Enhancing the dispatchability of distribution networks through utility-scale batteries and flexible demand, Energy and Buildings 172 (2018).

[63] F. Sossan, A. M. Kosek, S. Martinenas, M. Marinelli, H. W. Bindner, Scheduling of domestic water heater power demand for maximizing pv self-consumption using model predictive control, in: 2013 4th IEEE PES Innovative Smart Grid Technologies Europe, IEEE, 2013.

[64] R. Luthander, J. Widén, D. Nilsson, J. Palm, Photovoltaic self-consumption in buildings: A review, Applied Energy 142 (2015).

[65] E. Stai, F. Sossan, E. Namor, J. Y. L. Boudec, M. Paolone, A receding horizon control approach for re-dispatching stochastic heterogeneous resources accounting for grid and battery losses, Electric Power Systems Research 185 (2020).

[66] M. Kraning, Y. Wang, E. Akuiyibo, S. Boyd, Operation and configuration of a storage portfolio via convex optimization, in: Proceedings of the 18th IFAC World Congress, volume 18, 2011, pp. 10487-10492. 
[67] C. Bauer, S. Hirschberg, Potentiale kosten und umweltauswirkungen von stromproduktionsanlagen, PSI-Paul Scherrer Institut, Bundesamt für Energie BFE (2017) $1-10$.

[68] E. Namor, F. Sossan, R. Cherkaoui, M. Paolone, Control of battery storage systems for the simultaneous provision of multiple services, IEEE Transactions on Smart Grid 10 (2018) 2799-2808. 